\title{
A Population-based PBPK Model for the Prediction of Time-Variant Bile Salt Disposition within GI Luminal Fluids
}

\author{
Konstantinos Stamatopoulos* ${ }^{\dagger}$, Shriram M. Pathak ${ }^{\dagger}$, Luca Marciani ${ }^{\dagger \dagger}$ and David B. Turner ${ }^{\dagger}$ \\ ${ }^{\dagger}$ Certara Ltd (Simcyp Division), Level 2-Acero, 1 Concourse Way, Sheffield, S1 2BJ, United Kingdom \\ ${ }^{\dagger \dagger}$ Nottingham Digestive Diseases Centre and National Institute for Health Research, Biomedical Research Unit, Nottingham \\ University Hospitals, University of Nottingham, Nottingham, United Kingdom
}

\begin{abstract}
In vivo studies have shown cyclic bile salt (BS) outputs during fasting whereas higher amounts have been observed in fed states. This leads to fluctuations of intestinal BS concentrations ([BS]) that can affect the rate and extent of absorption of lipophilic drugs in particular. However, most PBPK models use fixed values of [BS] in fasted and fed states albeit with different values in different regions of the GI tract. During fasting, there is a relationship between gallbladder volume (GBV) and the phase of the Interdigestive Migrating Motor Complex cycle (IMMCc), showing cyclic GBV changes with periodic filling and emptying. This relationship is also affected by the origin of the IMMCc (antral or duodenal). In fed states, meta-analysis indicated that GB residual volume (\% of fasting maximum) was $46.4 \pm 27 \% \mathrm{CV}$ and $30.7 \pm 48 \% \mathrm{CV}$ for low- and high-fat meals respectively. The corresponding values for the duration of the emptying phase were for low fat meals $0.72 \mathrm{~h} \pm 1 \% \mathrm{CV}$ and for high fat meals $1.17 \mathrm{~h} \pm 37 \% \mathrm{CV}$. The model, the Advanced Dynamic Bile Salt Model (ADBSM), was built bottom-up and its parameters were not fitted against in vivo measurements of [BS]. It involved update of the dynamic luminal fluid volumes model based on meta-analysis of available imaging data. The ADBSM is incorporated into the Simcyp ${ }^{\circledR}$ (v18r2) PBPK simulator. The model predictivity was good (within 1.25 fold error for 11/20 of the clinical studies) and was assessed against clinical studies of luminal [BS] that provide only the type of meal (i.e., low- or high-fat), the timing of the meal and/or water intake events, and the number and age range of the study participants. The model is also an important component of models capturing enterohepatic recirculation of drug and metabolite. Further work is required to validate the current model and compare to simpler models with respect to drug absorption, especially of the lipophilic compounds.
\end{abstract}




\section{INTRODUCTION}

Along with other parameters, such as stability, permeability and first-pass metabolism, solubility in luminal fluids is considered to be a key factor for the prediction of oral drug bioavailability. Bile salts (BS) play a crucial role in the solubilisation and absorption of lipophilic and poorly soluble compounds. ${ }^{25-27}$ Several studies have been conducted in the past to characterize fasting and fed duodenal, jejunal and colonic aspirates in healthy ${ }^{2,27-31}$ and non-healthy (e.g., with ulcerative colitis) ${ }^{32}$ subjects. These studies have been used to design simulated biorelevant media (FaSSIF, FeSSIF) enabling in vivo prediction of drug solubility. ${ }^{33}$ Similarly, this information has been implemented in in-silico platforms; e.g., SimCYP, as a part of the Advanced Dissolution Absorption and Metabolism (ADAM) model ${ }^{34}$, GastroPlus and GISIM. ${ }^{35}$ However, BS concentrations ([BS]) used in in vitro dissolution experiments and in PBPK modelling are fixed and represent a snapshot of the dynamically changing in vivo environment. Furthermore, the in vivo information on [BS] integrated into in vitro and in- silico models, doesn't currently take into account biliary motility when aspirating duodenal and/or jejunal fluids. For instance, [BS] varies significantly within the GI tract due to a number of factors such as cyclic motility patterns of the gallbladder (GB) linked to the phase of the IMMCc, ${ }^{13,36,37}$ dynamic intestinal water volumes changes ${ }^{38,39}$ and postprandial strong GB contractions emptying a significant amount of BS. ${ }^{5,19}$ Furthermore, several studies have shown that in the fed state, the GB ejects $30-70 \%$ of its content within $20-30$ mins, depending on meal composition. ${ }^{4,8,39,40}$ Effort has been put into the development of mechanistic models incorporating biliary motility with the main focus on the GB. Guiastrennec et al., ${ }^{41}$ developed a mechanistic model of GB emptying in response to calorie intake with the intention to use it for predicting postprandial changes in drug absorption. However, the Guiastrennec et al., ${ }^{41}$ mechanistic model describes GB motility only in the fed state and is based on a single in vivo study. ${ }^{40}$ A different approach for mechanistically modelling GB motility is to link it to the secondary peaks appearing in the plasma concentration profile of drugs undergoing enterohepatic circulation (EHC). Abbiati and Manca $^{42}$ developed a PBPK model which links the GB emptying period with the onset time of secondary peaks in plasma concentration of sorafenib. However, this approach is essentially fitting the GB motility to reproduce the observed secondary peaks and does not take into account the different factors affecting/controlling GB motility. In other words, it may not be appropriate to link GB motility exclusively to the onset time of a secondary peak in the drug's plasma concentration profile; secondary peaks have appeared in cholecystectomized patients as well as in rats which do not have a gallbladder. The same approach has been taken by Jain et al. ${ }^{43} \mathrm{~A}$ more physiologically relevant mechanistic model of EHC has been developed by Lehr et al ${ }^{44}$ with the introduction of a clock time function as a control element of a trigonometric GB release function mimicking an ultradian rhythm. However, interindividual variability was applied only to the clearance and the distribution volume of the targeted drug (tesofensine). MRI and ultrasound measurements of fasting GB volume indicate high inter-subject variability. ${ }^{19}$ Furthermore, the GB volume changes linked to the IMMC are too complex ${ }^{22}$ to be described by a simple sine function. ${ }^{44}$ Another limitation of the Lehr et al ${ }^{44}$ approach is that the ultradian rhythm of the GB is linked to (i.e., starts at) the time of dose administration. However, dose can be administered randomly with respect to filling-emptying cycles of the GB which means, for example, that the GB won't necessarily enter an emptying phase due to drug administration. So, in order to be able to adequately predict the duodenal [BS] in fasted and fed states, GB motility ideally should be directly modelled based on the physiological parameters affecting it.

Thus, the present study aims to develop a more generalized mechanistic model of biliary system motility with the main focus on the GB, reflecting current knowledge obtained by a systematic literature review and meta-analysis. Thus, specific relationships between GB motility, residual volumes, bile secretion rates and gut motility in the fasting and fed states, are defined in order to build the mechanistic model. A general aim of the proposed mechanistic model is for it to be as flexible as possible in order to be easily coupled to EHC of different drugs and their primary and secondary metabolites. Furthermore, the proposed mechanistic model is coupled with the previously developed and recently updated Dynamic Fluid Volume 
model, as part of ADAM platform in SimCYP, to account the time-dependent intestinal [BS]. It is essential to use as accurate as possible luminal fluid volumes in order to simulate accurate [BS].

\section{METHODS}

Prior to model development, a systematic literature review and meta-analysis of the selected articles was conducted using PubMed and Scopus search engines. Reviews focused on th biliary system ${ }^{37,45-47}$ were screened first to gain a general idea about biliary motility and the factors affecting its functionality in fasting and fed conditions. Useful articles were manually extracted from those reviews and screened for eligibility. Then, the literature review focused first on GB motility and its relationship to gastroduodenal motility in the fasted state and thereafter to postprandial periods. The terms, "gallbladder", "gallbladder emptying", "gallbladder volume" and "Interdigestive Migrating Motor Complex" (IMMC) were used for the fasting state whereas "postprandial gallbladder motility" and "postprandial gallbladder volume" were used for the fed state. Search results were restricted to full text articles written in the English language, including healthy human subjects, of adult Caucasians. Additional articles were found through the manual search of included studies' references. Apart from the general criteria, the analytical methods used to monitor gallbladder motility in fasting and fed were critically evaluated and used as an inclusion or exclusion criterion, considering the opinion of the experts in the field, as it has been reported in the literature. ${ }^{6,48}$

Inclusion criteria:

1. Studies including healthy Caucasian subjects older than 18 years.

2. Studies using a physiological stimulus (i.e. meal) for postprandial gallbladder motility examination and giving details about the kcal and composition of the testing meal.

3. Studies using real-time ultrasonography and/or continuous scintigraphy as well as other validated imaging techniques like MRI.

Exclusion criteria:

1. Studies with pediatric age subject or animals.

2. Studies performing scintigraphy with single $i . v$. injection of radiotracer.

3. Studies where hormones like Cholecystokinin (CCK) and motilin were used to stimulate gallbladder and gastroduodenal motility.

In addition, literature review and meta-analyses were conducted for hepatic biliary secretions in fasted and fed conditions where, especially for the fed, only studies using physiological stimulus (i.e., a meal) were included. Furthermore, perfusion studies which provide data for the absorption of bile salts in different regions of the GI tract were also included.

Furthermore, papers providing individual profiles rather than only average profiles were mainly used to support the development of the model. The corresponding authors were contacted to provide the raw data from clinical studies under the new GDPR rules.

\section{RESULTS}

An initial screening showed 4,129 studies which potentially could be included (Fig. 1). After reviewing the title and abstract, 689 studies were selected for further analysis. Then 644 studies were excluded according to exclusion criteria as described in the methodology. Finally, the remaining 26 articles were subdivided into three groups, fasting, fed and human BS perfusion studies (3 articles). Fasting and fed 
groups were further divided into two subgroups: fasted-GB ( 7 articles), fasted-BS ( 3 articles), fed-GB (10 articles) and fed-BS (3 articles).

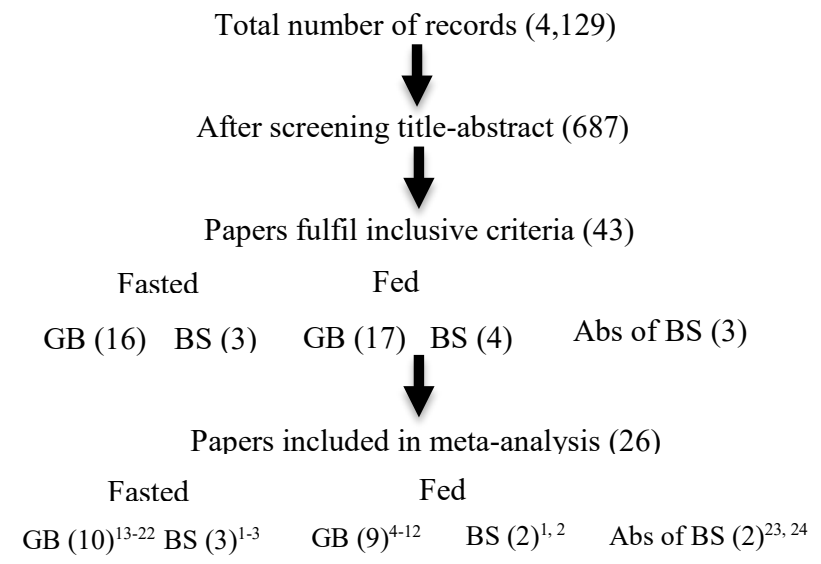

Fig. 1. Flow chart of the strategy used to select relevant studies. The literature search was done according to the guidelines of Preferred Reporting Items for Systematic Reviews and Meta-analysis (PRISMA); Gallbladder (GB), Bile Salts (BS), Absorption (BS)

\section{Fasting}

Various studies have shown strong correlation between the cyclic GB motility (i.e., repeating emptying and filling phases) and the IMMC ${ }^{13,22}$. Unlike the filling phase, the residual GB volume and the duration of the emptying phase depend on the origin of the IMMCc (gastric or duodenal). In particular, GB filling starts at phase I of the IMMCc with the GB reaching its maximum volume at $30 \%$ of the IMMCc cycle ${ }^{13}$, 22 . Thereafter emptying takes place which ends at $90 \%$ of the IMMCc, where phase III starts is of antral origin, or at $60 \%$ when phase III is of duodenal origin. ${ }^{13}$ Thereafter, a steady period, i.e. no further decrease or significant increase in the GB volume, takes place. Converting the GB fraction vs IMMC fraction relationship to actual $\mathrm{GB}$ volume $(\mathrm{mL})$ and IMMC duration time $(\mathrm{h})$, individual fasting $\mathrm{GB}$ volume change profiles can be generated with both intra- and inter-subject variability. This means that each Filling-Emptying (F-E) cycle is not the same within an individual's GBV profile. The generation of an individual $24 \mathrm{~h}$ GB profile is performed as follows:

1) Individual maximum GB volume (mGBv): in order to calculate GBV fraction at each IMMC time point, the mGBv per individual is needed. The $\mathrm{mGBv}(\mathrm{mL})$, which is taken to represent the maximum anatomical GB volume, is considered the maximum volume reported in the studies used in the meta-analysis. Normally fasting GB volume is measured 5, 10, 15 min or more before meal. For those studies which provide only the mean value of those measurements per individual is used as "the anatomical GB volume". However, for those studies reporting all the measurements, only the maximum value is selected. To generate an individual $\mathrm{mGBv}$, random sampling is performed from a normal distribution with mean $=18.8 \mathrm{~mL}, \% \mathrm{CV}=53$, and range $=4.0-93.0 \mathrm{~mL}$ (Table 1 ).

2) Number and duration of IMMC events per individual: now the \%IMMC time points need to be converted to actual time (h). First it should be pointed out that the number $(N)$ of F-E cycles and the duration of each $N^{\text {th }}$ cycle should be linked to the number of IMMC events observed in an individual 
within a $24 \mathrm{~h}$ period time as well as to the duration of each IMMC. Furthermore, the duration of each IMMC depends on the origin (i.e. antrum or duodenum). Although, IMMC can start from other origins, i.e. esophagus, jejunum ${ }^{49}$, however, limited info was found with regards to fasting GB volume vs IMMC profile, in particular in jejunum. ${ }^{50}$ Hence, the meta-analysis was focused on IMMC events started from antrum and duodenum. So, the percentage (i.e. the probability) of the IMMC events started from antrum was obtained by dividing the number of IMMC events commencing for example in the antrum with the total number of IMMC events commencing in both regions, antrum and duodenum. Thus, the probability of the IMMC starting from the antrum is $60 \%$ and $40 \%$ from duodenum. Then, a random number between $0-1$ is generated from a uniformly distributed random numbers. If this number is $<0.6$, then the first IMMC event is assigned to antrum and hence the duration (h) of this IMMC event is randomly generated from normal distributed data with mean $=2.6, \% \mathrm{CV}=$ 30.2 , and range $=0.9-4.3$ (Table 1). Otherwise the IMMC is assigned to duodenum with mean=1.3, $\% \mathrm{CV}=60.3$ and range $=0.5-3.4$. The same procedure was repeated for the next IMMC and until the cumulative sum of the duration of the IMMC events is in total $\leq 24 \mathrm{~h}$ for every individual.

3) Obtaining filling and emptying rate constants for each F-E cycle of each individual: knowing the individual $m G B v$ and the duration of each IMMC, the corresponding GB volumes at 60 and $90 \%$ of each IMMC can be obtained. In particular, a random \%GBV value is generated from normal distributed data with mean $=74.0, \% \mathrm{CV}=10$, and range $=70-100$, in case where the IMMC starts from antrum, and mean $=80.7, \% \mathrm{CV}=17$, and range $=70-100$, if IMMC starts from duodenum. This $\% \mathrm{GBV}$ is the $\%$ residual GB volume at $90 \%$ of IMMC or at $60 \%$ of IMMC,

Table 1. Values of the parameters used in the model to generate individual gallbladder volume changes profile as well as the active and passive absorption components of BA in GI tract segments.

\begin{tabular}{|c|c|c|c|c|}
\hline Parameter name & PopRep* & $\% \mathrm{CV}$ & Min & Max \\
\hline IMMC Cycle (Antral Origin) (h) & 2.6 & 30.2 & 0.9 & $4 \cdot 3$ \\
\hline IMMC Cycle (Duodenal Origin) (h) & 1.3 & 60.3 & 0.5 & $3 \cdot 4$ \\
\hline Probability of IMMC Cycle Originating in the Antrum (\%) & 60 & NA & o & 100 \\
\hline Probability of IMMC Cycle Originating in the Duodenum (\%) & 40 & NA & 0 & 100 \\
\hline Maximal Gallbladder Volume (mL) & 18.8 & 53 & 4.0 & 93.0 \\
\hline Fasted Gallbladder Residual Volume (\%)- IMMC of Antral Origin & 74.0 & 10 & 70.0 & 100 \\
\hline Fasted Gallbladder Residual Volume (\%)- IMMC of Duodenal Origin & 80.7 & 17 & 70.0 & 100 \\
\hline Gallbladder Residual Volume (\%) Fed, High-Fat & 30.67 & 48 & 3.70 & $99 \cdot 50$ \\
\hline Gallbladder Residual Volume (\%) Fed, Low-Fat & 46.36 & 27 & 27.0 & 79.2 \\
\hline Duration of Gallbladder Emptying Period (h), High-Fat & 1.17 & 37 & 0.33 & 2.08 \\
\hline Duration of Gallbladder Emptying Period (h), Low-Fat & 0.72 & 1.18 & 0.717 & 0.733 \\
\hline
\end{tabular}




\begin{tabular}{|c|c|c|c|c|}
\hline Pappcoef for Jejunum I-II (mmol/h/mM) & 0.42 & 14 & 0 & 0.54 \\
\hline Jmax for lleum I-IV $(\mathrm{mmol} / \mathrm{h})^{b}$ & 2.48 & 20 & 0 & 3.49 \\
\hline Km for lleum I-IV (mmol)c & 0.6 & 33 & 0 & 1 \\
\hline Pappcoef for Colon $(\mathrm{mmol} / \mathrm{h} / \mathrm{mM})^{a}$ & 0.0565 & 30 & 0 & 1 \\
\hline $\mathrm{f}_{\mathrm{BS} \text { abs }}$ Jejunum I-II & 0.15 & NA & 0 & 1 \\
\hline $\mathrm{f}_{\mathrm{BSabs}}$ Ileum I-II & o & NA & o & 1 \\
\hline $\mathrm{f}_{\mathrm{BS} a b s}$ Ileum III-IV ${ }^{d}$ & 0.05 & NA & o & 1 \\
\hline $\mathrm{f}_{\mathrm{BSabs}}$ Colon $^{d}$ & 1 & NA & o & 1 \\
\hline BS Weighted Avg. MWt. & 400 & - & 100 & 1000 \\
\hline Fasted-HBS massrate $(\mathrm{mmol} / \mathrm{h})^{\mathrm{e}}$ & 0.19 & 69 & 0.12 & 0.28 \\
\hline Fed-(HBS massrate $)(\mathrm{mmol} / \mathrm{h})^{\mathrm{e}}$ & 1.33 & 45 & 1.33 & 1.44 \\
\hline$\%$ of Hepatic Bile Entering Gallbladder & 70.3 & 32.1 & 0 & 100 \\
\hline
\end{tabular}

${ }^{\mathrm{a}}$ Pappcoef $=$ Apparent permeability coefficient $(\mathrm{mmol} / \mathrm{h} / \mathrm{mM}) ;{ }^{\mathrm{b}} \mathrm{Jmax}=$ Maximum Rate of Bile Absorption $(\mathrm{mmol} / \mathrm{h}) ;{ }^{c} \mathrm{Km}=$ Michaelis-Menten constant $(\mathrm{mmol}) ;{ }^{\mathrm{d}} \mathrm{f}_{\mathrm{BSabs}}=$ Bile salt fraction available for absorp-

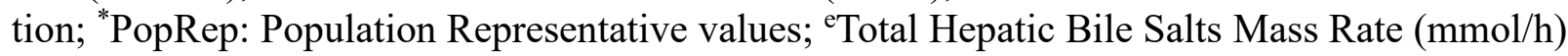

according to the origin. In addition, this first generated \%GBV value is used as the initial condition $(\mathrm{t}=0)$ for generating the individual $24 \mathrm{~h}$ fasting GB volume changes profiles. As mentioned above the $m G B v$ is normally observed at $30 \%$ of the IMMCc. Thus, it is assumed that the GB volume at $30 \%$ of each IMMCc, 
always corresponds to the $m G B v$. This is in accordance with the observed data where the GB's filling phase continues, unless meal is given, until the mGBv has been reached and thereafter a new emptying phase will occur. $^{22,51}$ It is also assumed that the filling phase takes place is zero order $(\mathrm{IMMCc}=0$ $-30 \%$ ), whereas the emptying phase under first order kinetics ${ }^{52}$ followed by a constant volume. The filling (Eqs. 1-2) and emptying (Eqs. 3-4) rate constants used to generate the individualized $24 \mathrm{~h}$ GBV fasting profile are showed below.

$k_{f i l_{-} a_{i-N}}=\frac{m G B v_{i}-m G B v_{i} * G G B V_{@ 90 \% I M M C_{N}}}{0.3 * \operatorname{DurIMMC}_{N}}(\mathrm{~mL} / \mathrm{h})$

$k_{f i l_{-} d_{i-N}}=\frac{m G B v_{i}-m G B v_{i} * \% G B V_{@ 60 \% I M M C_{N}}}{0.3 * \operatorname{DurIMMC}_{N}}(\mathrm{~mL} / \mathrm{h})$

$k_{\text {em_a } a_{i-N}}=\frac{\ln \left(m G B v_{i}\right)-\ln \left(m G B v_{i} * \% G B V_{@ 90 \% I M M C_{N}}\right)}{0.6 * \operatorname{DurIMMC}_{N}}$

$\left(\mathrm{h}^{-1}\right)(3)$

$k_{\text {em_d } d_{i-N}}=\frac{\ln \left(m G B v_{i}\right)-\ln \left(m G B v_{i} * \% G B V_{@ 60 \% I M M C_{N}}\right)}{0.3 * D u r I M M C_{N}}$ $\left(\mathrm{h}^{-1}\right)$ (4)

Where the letters ' $a$ ' and ' $d$ ' denote the origin of the IMMCc ('a'=antral and ' $d$ '=duodenal) whereas DurIMMC denotes the actual duration of the $N^{\text {th }}$ IMMC (h). After obtaining the F-E constants for $1^{\text {st }}, 2^{\text {nd }}, \ldots, N^{\text {th }}$ IMMC assigned to $1^{\text {st }}$, $2^{\text {nd }}, \ldots, i^{\text {th }}$ individual, the following equations were used for the filling (Eq. 5), emptying (Eq. 6) and steady period (Eq. 7) to obtain all the data points for the $24 \mathrm{~h}$ fasting profile of GBV.

$\frac{d G B V}{d t}=k_{f i l_{a_{i-N}}}, \frac{d G B V}{d t}=k_{f i l_{d-N}}$

$\frac{d G B V}{d t}=-k_{e m_{a_{i-N}}} * G B V$,
$\frac{d G B V}{d t}=-k_{e m_{d_{i-N}}} * G B V$

A
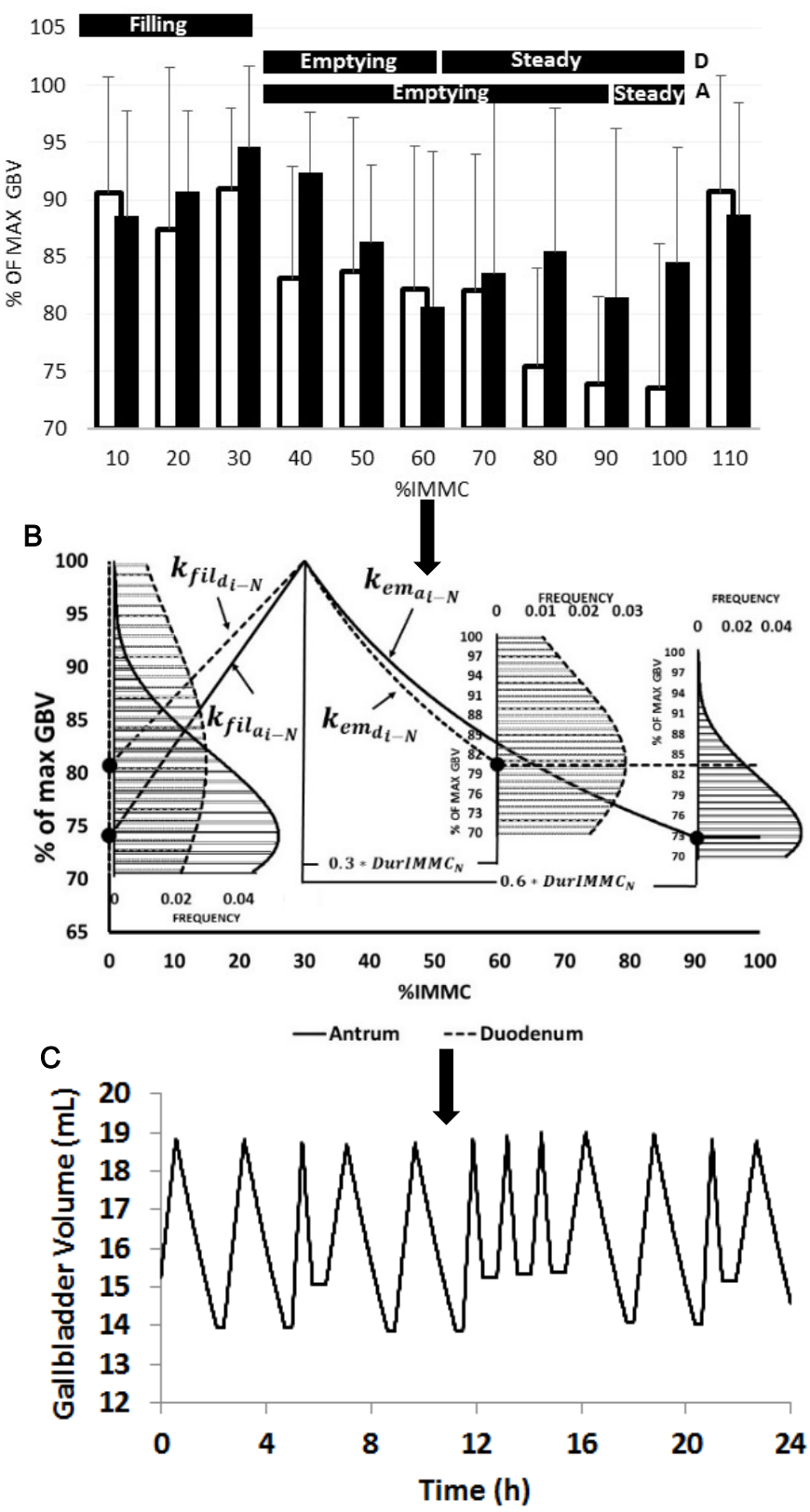

$\frac{d G B V}{d t}=0$

Fig. 2 shows how the observed relationship between \%GBV and \% IMMC is converted to individual GBV (mL) vs IMMC (h) profile using Eqs. 1 - 7 after random generation of $\mathrm{mGBv}$, origin and duration 
of each IMMC per individual and $\% \mathrm{GBV}$ at $\mathrm{t}=0$ (only once per individual), $60 \%$ or $90 \%$ depending on the origin of each IMMC. Fig. $2 \mathrm{C}$ shows clearly the difference between E-F cycles which associated with IMMCc of duodenal and antral origins, in terms of decrement of GBV and duration of emptying phase.

Fig. 2. Generation of $24 \mathrm{~h}$ individual fasting GBV profiles. A) Relationship between \%GBV and \%IMMC with respect to the origin of the IMMC. The horizontal solid bars indicate the different GB FE phases with respect to the IMMC for both origins, D:duodenal and A:antralm (modified from Stolk et al $1993^{13}$ ); B) An example showing how a \%GBV vs. \%IMMC profile is created for one IMMC cycle from in vivo data (A) after generating random $\% \mathrm{GBV}$ numbers sampled within the specific range of the normal distribution curves at $60 \%$ of IMMC for those IMMCs originating in the duodenum and at $90 \%$ of IMMC for those IMMCs of antral origin; at $\mathrm{t}=0$, random sampling performed only once per individual; C) An example of a $24 \mathrm{~h}$ fasting GBV profile of a representative (average) individual with $\mathrm{mGBV}=18.8$ (mL), 12 IMMC events where 7 starting from antrum and 5 from duodenum with a sequence as followed: A A D A A D D D A A D A

\section{Fed}

Strong contractions of the GB occur upon stimuli such as after meal ingestion. Depending on the strength of the stimulus, e.g. fat content of the meal, the residual GBV and the duration of the emptying phase vary significantly. Clinical studies passing the inclusion criteria were split to two groups based on the \%fat caloric content contributing to the total kcal of the meal. Thus, a high-fat meal is defined when the $\% \mathrm{kcal}$ coming from fat is $\geq 40 \%$. Meta-analysis showed that the duration of the GB emptying phase is for 0.72 $\pm 30(\% \mathrm{CV}) \mathrm{h}$ (range: $0.5-0.73 \mathrm{~h})$ and $1.17 \pm 37(\% \mathrm{CV}) \mathrm{h}$ (range: $0.33-2.08 \mathrm{~h})$ for low- and high - fat meals respectively $(\mathrm{p}<0.05)$. After the emptying period, the GB enters the refilling phase, treated as zero order as indicated previously, until reaching the maximum ('anatomical') volume, ${ }^{5}$ unless interrupted by further food intake. ${ }^{53}$ Based on our knowledge and the available data in the public domain, there are not enough prolonged recordings of the GB motility to show if the GB enters the fasting F-E cycling profile exactly after the postprandial refilling period. Thus, it's assumed that after GB reaches the maximum volume, a new fasting F-E cycling pattern is followed which continuous until the next meal. Furthermore, the common practice to investigate GBV changes and duodenal [BS] under multiple meals is to administer food every 4, 5 and/or $6 \mathrm{~h} .{ }^{1,53-55}$ However, it was not where possible to find information indicating how the GB behaves with shorter time intervals between the meals (e.g. every 1, 2, $3 \mathrm{~h}$ etc.). Nevertheless, 1 or $2 \mathrm{~h}$ time interval between meals might be too short reflecting a situation where the GB would be physiologically inactive due to continuous feeding. ${ }^{54}$ Thus, the proposed model allow the GB to enter a fed state only if the next meal is $4 \mathrm{~h}$ after the previous one, otherwise the GB's refilling phase continues until mGBv has been reached.

Generation of the individual fed GBV profile is performed as follows: A meal can be given at random time point during an individual IMMCc and thus at a random point dur-ing the GB cyclic fasting profile; the meal, of course, interrupts the fasting profile. Thus, the fasting GBV (taken from the pre-generated fasted GBV profile) at the time of interruption $\left(t_{1}\right)$ is used as the starting point for the emptying phase of the GB in the fed state $\left(G B V_{@ t_{1}}\right)$. Fed state GB emptying is characterized by a first order process (Eq. 8).

$$
\frac{d G B V}{d t}=-k_{G B e m f e d} * G B V_{@ t_{1}}
$$

where

$k_{\text {GBemfed }}=\frac{\ln \left(G B V_{@ t_{1}}\right)-\ln \left(G B V_{@ t_{2}}\right)}{t_{e m}}\left(h^{-1}\right)$

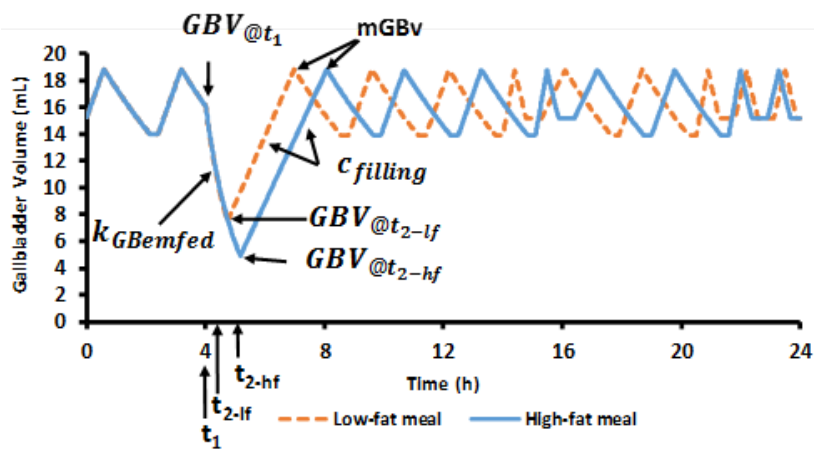


$t_{2}=t_{1}+t_{e m}(h)$

$G B V_{@ t_{2}}=\left(G B V_{@ t_{1}} * \operatorname{ResVol}_{\%}\right) / 100(\mathrm{~mL})$

ResVol\%=\%Residual Volume of GBV@t 1 which is randomly generated from normally distributed data with mean $=30.67, \% \mathrm{CV}=48$, and range $=3.7-99.5$ for high-fat meals or, for low fat meals, mean $=40.36$, $\% \mathrm{CV}=27$, and range $=27-79.2$.

To generate individual fed GBV profiles, a transition from the emptying to refilling phase is needed. Meta-analysis showed that there is a linear correlation $\left(\mathrm{R}^{2}=0.6, \mathrm{SF} 12\right)$ between the emptying rate constant $k_{\text {GBemfed }}\left(\mathrm{h}^{-1}\right)($ Eq. 9$)$ and the refilling rate $c_{\text {filling }}(\mathrm{mL} / \mathrm{h})$, regardless of the fat content of the meal. Linear regression of the data gave Eq. 10 which was used in Eq. 11 to obtain the GB refilling profile. Thus, after

obtaining $k_{\text {GBemfed }}$, Eq. 10 is used to calculate $c_{\text {filling. }}$. Then Eq. $8(\mathrm{t} 1 \leq \mathrm{t}<\mathrm{t} 2)$ and Eq. 11 (GBV@t2- $i \leq$ $\mathrm{GBV}<\mathrm{mGBv}, i=l f$ or $h f$ for a low fat or high fat meal respectively) are used to generate the fed GBV profile.

$$
\begin{aligned}
& c_{\text {filling }}=4.7363 * k_{\text {GBemfed }}(\mathrm{mL} / \mathrm{h}) \\
& \frac{d G B V_{\text {filling }}}{d t}=c_{\text {filling }}
\end{aligned}
$$

Fig. 3 shows an example of a fasting-fed-fasting GBV profile for low- and high-fat meals. The GBV fasting profile prior to the meal corresponds to the fasting GBV profile as showed in Fig. 2C. After the end of refilling phase the GB enters the fasting profile which is re-generated as described in the fasted section for the remaining time period. This example illustrates the effect of a high-fat meal compared to a low-fat meal where normally a higher fraction of the stored bile is ejected from the GB into the duodenum and the duration of the refilling phase is longer; $\sim 3 \mathrm{~h}$ compared to $\sim 1.5 \mathrm{~h}$ for a low-fat meal.

Fig. 3. Example of fasting - fed - fasting transitions; a scenario where meal is given at a random time point of the 24 h GB volume profile; GBV@t2-lf: residual GBV after low-fat (lf) meal ingestion; GBV@t2-hf: residual GBV after high-fat (hf) meal ingestion; t1: random time-point of meal administration; t2-lf: time point at which the emptying phase ends after low-fat meal; t2-hf: time point at which emptying phase ends after high-fat meal.

\section{Bile Salt Disposition within GI Luminal segments}

Knowing the GBV profile, either fasting and/or fasting-fed-fasting, the fluctuating duodenal, jejunal, ileal and colonic bile salts -times concentrations profiles- $(\mathrm{mM})$ can be obtained. However, it is also important to know hepatic bile salt secretions in the fasted and fed states, time-dependent water volume distribution and absorption kinetics within the different GI tract segments. The ADAM model in Simcyp simulator (v18r2) provides nine luminal segments (stomach, duodenum, jejunum 1-2, ileum 1-4 and colon) with a dynamic water volume distribution in each segment, transit rate constants, dynamic (i.e., time varying) secretions (saliva, gastric and intestinal secretions such as from the Brunner's glands) as well as absorption rate constants for water. Furthermore, within this work, passive and active absorption of bile salts in the different ADAM GI tract segments was modelled based on absorption kinetics derived from 
human perfusion studies. ${ }^{23,24}$ Based on the available data, passive absorption of BA predominates in jejunum 1-2 and the colon whereas active absorption predominates in the ileal segments. For the passive component in jejunum 1-2, glycochenodeoxycholic acid (GCDC) was used as reference ${ }^{23}$ and chenodeoxycholate (CDCA) in colon $^{24}$ whereas taurocholate (TA) was used as reference for active absorption in the ileal segments. ${ }^{23}$ However, TA, GCDC and CDCA are only a fraction of the total BA present in luminal fluids, hence the fraction available for passive absorption in the jejunum and colon as well as active absorption in ileum, should be assigned to the fraction of TA, GCDC and CDCA present in lumen aspirates. ${ }^{27}$ However, differences in the luminal bile salts compositions have been found between studies, which can be attributed population differences. ${ }^{2}$ In this work, the bile salt composition, as published by Riethorst et al (2016), ${ }^{2}$ was used as a case study based on which fractions of GCDC and TA were found to be $30 \%$ and $10 \%$, respectively. Thus, for the passive component the fraction available was set to 0.15 for each jejunal segment. The active component was set to 0.05 for ileum $3 \& 4$ and zero for ileum $1 \& 2$, since the perfusions studies have been conducted mainly within the terminal ileum (i.e. within $30 \mathrm{~cm}$ of the ileocecal valve). The passive component was set to zero for all ileal segments, as active absorption takes place mainly within this region.

Eqs 12 - 18 were used to obtain the time-dependent biliary secretions following the F-E phases of the pre-generated GBV profile:

\section{- $\quad$ Filling period of GB}

Hepatic bile secretions $(\mathrm{Hsr}, \mathrm{mL} / \mathrm{h}$ ) are back-calculated from the filling rate constant of the GB. This is based on the fact that the liver is the only source of bile flow during the closure of the sphincter of Oddi and relaxation of the GB muscle tissue. Thus the GB filling rate should reflect the hepatic secretion rate, accounting also for the fraction of the bile entering the GB.

$H s r(t)=\frac{k_{f i l}}{f r_{\text {bile enter } G B}}$

where $k_{f i l}$ is derived from Eqs 1 and 2 and $f r_{\text {bile enter } G B}$ is the \% of Hepatic Bile Entering Gallbladder (Table 1).

- Bile flow rate to duodenum $(\mathrm{BfD}) \mathrm{mL} / \mathrm{h}$ :

$B f D(t)=H \operatorname{sr}(t) *\left(1-f r_{\text {bile enter } G B}\right)$

- Rate of bile salt entering the duodenum (BSenDuo):

BSenDuo $=H B S_{\text {massrate }-c} *\left(1-f r_{\text {bile enter } G B}\right)$

where $H B S_{\text {massrate }}$ is the hepatic bile salt mass rate, $\mathrm{mmol} / \mathrm{h}$ (Table 1$)$ and c $=$ 'fasted' or 'fed'.

- $\quad$ Emptying period of GB

○ Hsr, according to Eq. 12

$\circ \underline{B f D}$

$B f D(t)=H s r+k * G B V(t)$ 
where $k$ is the emptying rate constant $\left(\mathrm{h}^{-1}\right)$ in fasted or fed state and switches from one state to another according to the GBV profile.

- BSenDuo

$\operatorname{BSenDuo}(t)=H B S_{\text {massrate }-c}+k * B \operatorname{SinGB}(t)$

where $H B S_{\text {massrate }}$ and $k$ have the same function as described in Eqs. 14 and 15, respectively.

- $\quad$ Steady period of GB

This period is only applied during the fasted state.

○ Hsr, according to Eq. 12

$\circ \mathrm{BfD}$

$B f D(t)=H s r$

○ $\underline{\text { BSenDuo }}$

$\operatorname{BSenDuo}(t)=H B S_{\text {massrate }_{c}}$

Eqs. 19 - 21 were used to obtain time-variant luminal bile salt concentrations within the ADAM GI tract segments; bile salts concentration in stomach is static for healthy humans albeit with inter-subject variability.

$\frac{d B S m_{\text {Duo }}}{d t}=\frac{d B S e n D u o}{d t}-k t_{i} * B S m_{i}$

$\frac{d B S m_{J e j 1}}{d t}=k t_{i-1} * B S m_{i-1}-k t_{i} * B S m_{i}-K A b s_{i}$

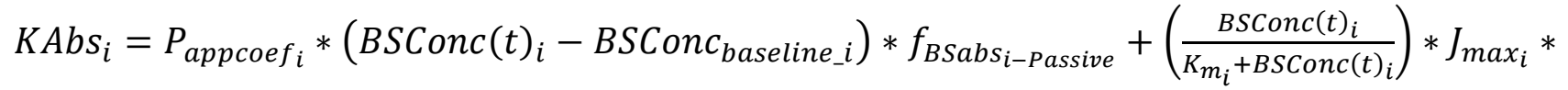

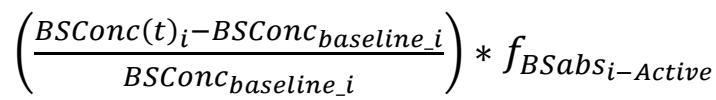

where $i$ indexes the duodenum, jejunum $1 \ldots$ colon, $B S m$ is the bile salt mass (mmol) in each segment, $K A b s$ is the absorption rate constant of bile salts. $f_{B S a b s_{i-P a s s i v e}}$ and $f_{B S a b s_{\text {-Active }}}$ is the fraction of bile salts available for absorption for the active and passive component, respectively. BSConc baseline $_{\text {in }}$ the [BS] baseline (i.e. steady state) in all ADAM segments based on prior meta-analysis. BSConc is the timedependent $[\mathrm{BS}](\mathrm{mM}) . k t$ is the transit rate constant $\left(\mathrm{h}^{-1}\right)$ derived from in-house meta-analysis of water volume distributions and transit in GI tract of healthy volunteers based on MRI and scintigraphy in vivo studies. Hence, the transit term, $k t\left(\mathrm{~h}^{-1}\right)$, "links" bile salt concentration from one segment to the other one. $P_{\text {appcoef }}$ is the apparent permeability coefficient $(\mathrm{mmol} / \mathrm{h} / \mathrm{mM})$, Jmax is the maximum rate of bile acid 
uptake $(\mathrm{mmol} / \mathrm{h})$ and $K m$ is Michaelis-Menten constant (mmol). The values of $P_{\text {appcoef, }}$ Jmax and $K m$ are shown in Table 1.

Eq. 21 shows that the passive and active absorption component is available for all ADAM segments but since, for example the passive component is considered to be zero in the ileum segment the first term of $K A b s$ will be zero and hence only the second (i.e., active uptake) will contribute to the total [BS] in ileum. However, this gives flexibility to apply both or only one absorption component according to new findings or data available for bile salt absorption in GI tract. The terms $\left(B S \operatorname{Conc}(t)_{i}-B_{S C o n c}\right.$ baseline $\left._{-}\right)$and $\left(\frac{\left.B_{S C o n c}(t)_{i}-B_{S C o n C_{\text {baseline }} i}\right)}{\text { BSConc }_{\text {baseline }} i}\right)$ (Eq. 21), prevent the $[\mathrm{BS}]$ to drop below BSConc baseline. In most subjects BSConc $_{\text {baseline }}>$ critical micelle concentration $(\mathrm{CMC})$ of bile salts in the fated as well as the fed state.

Eq. 22 is used to calculate the time-variant [BS] in GI tract each segments.

$$
B S_{\text {concen }_{i}}=\frac{B S m_{i}}{V_{i}(t)} * 1000(m M)
$$

where $\operatorname{Vi}(t)$ is the time-dependent fluid volume in the fasted or fed state in the $i^{t h}$ ADAM GI tract segment (as discussed below, first-order transit rate constants are used).

\section{DISCUSSION}

After constructing all the equations describing the time-variant EHC of bile salts coupled to time-dependent luminal fluid volumes, simulated luminal $[\mathrm{BS}]$ were obtained and compared to the observed data. The ADBSM was tested for its predictiveness with informing Simcyp ${ }^{\circledR}$ (v18r2) simulator only with the type of meal (i.e. low- or high-fat), the time of the meal intake and/or water intake events (using the Food Staggering Custom Dosing tool), the number, gender and age- range of the participants in each study. Thus, the ADBSM was not adjusted, optimized or tested against a single study. Moreover, it should be pointed out that none of the clinical studies selected for the performance verification (PV) provided information about GB volumes, transit rates, IMMC events and their duration, fluid volumes etc. In total 12 clinical studies were used for PV of the model (Table S1). Figs. 4, 5, 6 and 7 show the time-dependent [BS] in the different ADAM compartments, duodenum, jejunum I-II, ileum I \& IV as well as colon respectively, according to the region of the GI tract where samples collected in each study; the individual outputs of the ADBSM for all ADAM compartments for each in vivo study can be found in the supplementary material.

In Fig. 4A the simulated data were plotted against those reported by Yao et al 2002. The ADBSM was able to capture the range of the individual [BS] profiles with $95 \%$ confidence interval. The mean maximum [BS] of the observed and simulated data was $16 \pm 14 \mathrm{mM}$ and $13 \pm 14 \mathrm{mM}$, respectively, within the time period of the in vivo study; $15 \mathrm{~min}$ before a meal and $120 \mathrm{~min}$ after. Figs. 4A(a' - d') show the simulated individual profiles selected from the pool, with the best matching to the observed individual profiles. Unlike to the maximum [BS], where no significant differences were found between observed and simulated profiles ( $p=0.1, \mathrm{t}$-test), the values of the $[\mathrm{BS}]$ after $\sim 5 \mathrm{~h}$ were higher in observed data without showing further decline; see especially Individual 1 (Fig. 4Aa') and 2 and (Fig.4Ab'). If it's assumed that these steady state concentrations are related to the fasted [BS] period for those individuals, perhaps the main reason for higher fasted [BS] in some individuals, might be the baseline fluid volumes differences between the virtual populations and the healthy volunteers. 

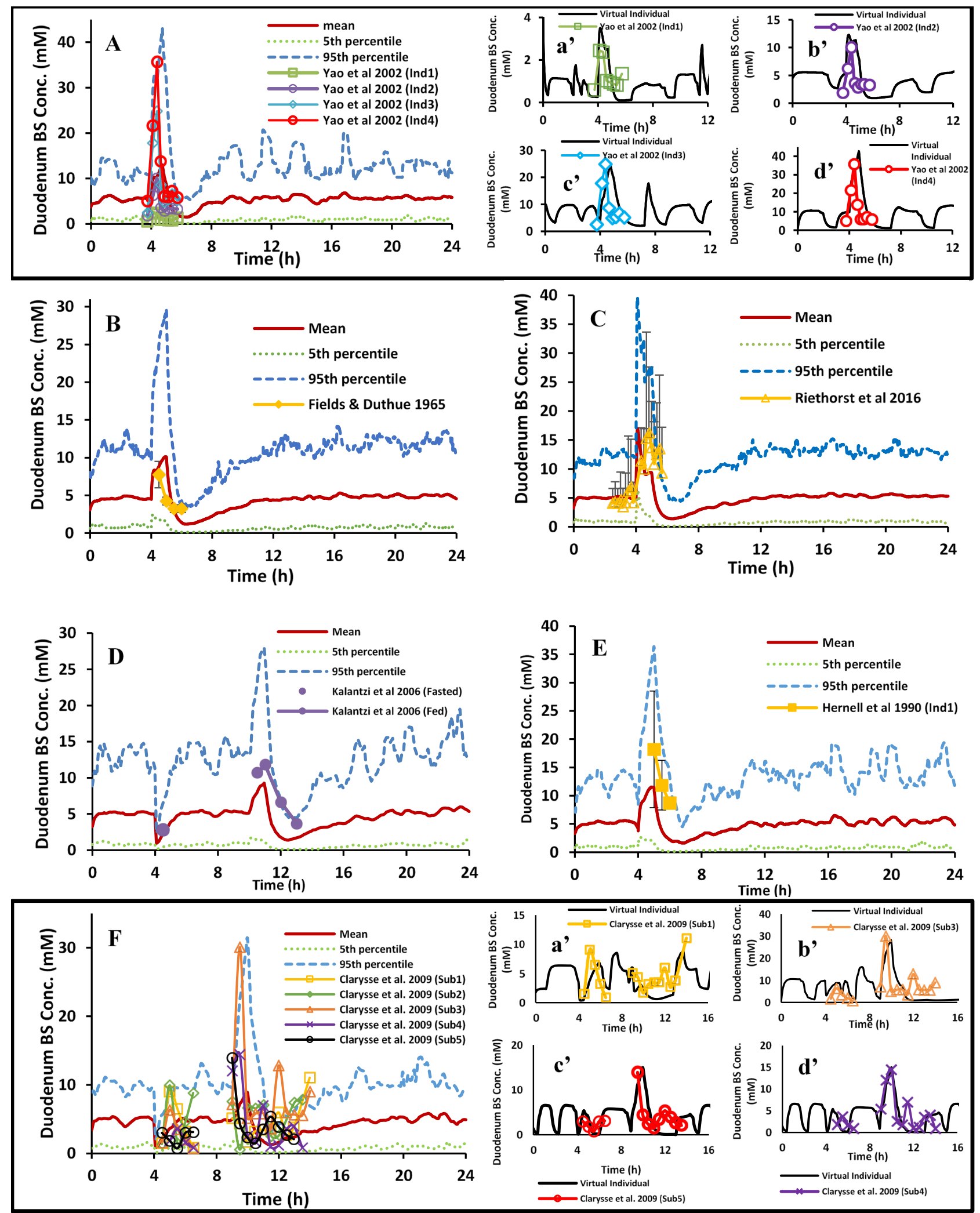

Fig.4.Time-dependent duodenal [BS] (BS Conc. $\mathrm{mM}$ ) of ADBSM in duodenum overlaid with in vivo observations. The simulation was performed in different states according to the protocol of the clinical study: A) fed, Yao et al 2002; B) fed, Fields \& Duthue 1965; C) fed, Riethorst et al 2016; D) fasted starting @4h followed by fed starting @10h, Kalantzi et al 2006; E) fed, Herenll et al 1990; F) fasted starting @4h followed by fed starting @9h, Clarysse et al 2009; (for details see supplementary material). 
Figs. 4B-E show PV of the model for the various studies which provided only the average [BS] vs time information. In these studies the average profile was within the predicted range of 5\%-95\% confident intervals. In fig.4B the maximum [BS] was $7.7 \mathrm{mM}$ at $4.5 \mathrm{~h}$ and $9.8 \mathrm{mM}$ at $4.9 \mathrm{~h}$ for observed and simulated data, respectively $(p=0.24)$. In observed data, fasted $[\mathrm{BS}]$ values were restored $1.5 \mathrm{~h}$ from meal ingestion without further change after $2 \mathrm{~h}$. In the simulated profiles there was a deeper decrease in [BS] whereas steady state fasted [BS] values were restored $7 \mathrm{~h}$ after a meal reaching similar values with the observed data. However the duration of the study is not long enough to know the evolution of [BS]. In fig. $4 \mathrm{C}$ the fasting values of [BS], i.e. before meal (2.5-3.8 h), were $4.7 \pm 1.05 \mathrm{mM}$ and $5.13 \pm 3.9 \mathrm{mM}$ for observed and predicted data $(p=0.12)$, respectively. In the fed period, i.e. $4.3-5.66 \mathrm{~h}$, the [BS] values were 12.6 $\pm 2.2 \mathrm{mM}$ and $7.3 \pm 7.9 \mathrm{mM}$ for observed and predicted data ( $p=0.001$, t-test), respectively. So, there is a statistical significant difference in the fed state between observed and simulated values. In the simulated profile the [BS] declines faster than the observed values although the latter show still elevated [BS]. Riethorst et al noted that the [BS] were not restored to fasted values after 90 min of sampling following a meal. In addition, the observed [BS] showed oscillations, i.e. repeated peaks (see SFig.9) in the fed state. The mean simulated profile cannot explain adequately these in vivo observations. However, it should be mentioned that the model can capture the large variability of the observed data (see SFig. 9A) for both fasted and fed state within the physiological range. Only one virtual individual showed in fed state very high [BS] values $(\sim 100 \mathrm{mM})$ which is comparable to the highest value of $86.4 \mathrm{mM}$ reported by Riethorst et al. In addition, in simulated profiles there is sharp increase in [BS], almost instantaneously upon meal ingestion. This is because, based on the model design, the GB responds immediately to a meal. However, it has been shown that there is a lag time between meal ingestion and GB emptying linked to the phase of IMMCc at which meal was given. ${ }^{58}$ However, there is only one study showing this and to the best of the current authors knowledge there is no other study investigating this phenomenon. It seems unlikely, although motility was not recorded in the Riethorst et al study, that in all subjects the meal was given at the same IMMCc phase something which could explain the delayed response of the GB and hence a shift to the maximum peak upon GB contraction. Although, there is no information for the first 30 minutes in the Riethorst et al study, it seems that this is the only study where duodenal [BS] are so high. Most of the studies (Figs. A, D, E and F) used for PV showed sharp increase and decrease of [BS] following the simulated profiles. This can be attributed to differences in the sampling time intervals and sample volume and hence how extensively the EHC has been interrupted, population differences in biliary secretions, fluid volumes, ${ }^{59} \mathrm{GI}$ tract motility and so on. With regard to the multiple peaks in the observed profiles, SFig. 9B shows three characteristic simulated profiles with one close to the observed [BS] duodenal profile. According to the selected virtual profiles, the subjects with the elevated multiple peaks should have GB kinetics which combine a deep decrease in GB volume upon meal ingestion followed by a more than $25 \%$ reduction of the maximum anatomical volume in the first fasting emptying phase appearing once the prolonged refilling phase in the fed state has been completed (SFig.9C, virtual individual162-Trial1). These GB kinetics lead to no significant accumulation of BS in the GB (SFig.9D). In addition, the subjects are expected to have relatively low duodenal fluid volumes (SFig.9E) combined with low fluid transit times (see supplementary materials), resulting in elevated duodenal [BS]. However, these conditions are for an average subject whereas individual profiles, if available, may tell a different story.

Prolonged measurements of duodenal [BS] as in the Clarysse et al 2009 study (Fig.4F (a'-d')) showed multiple peaks appearing in [BS] several hours after a meal. There may be several reasons for these secondary peaks. It may be due to the GB fasting emptying-filling cycle which may appear sooner than the simulated profile. However, this cannot be absolutely true because it takes several hours for the GB to enter its fasting profile as a prolonged refilling phase take places after the strong GB contraction of due to meal ingestion. ${ }^{60}$ This can be also seen from the mean simulated profile (Fig. 4F) where a rise of the [BS] and the following peaks were observed several hours after a meal. This deep drop in [BS] after the high peak (between $\sim 5-7.4 \mathrm{~h}$ ) is related mainly to GB kinetics where the GB enters the refilling phase, 
hepatic secretions are lower (i.e. back to fasting secretion rates) and to the dilution of the duodenal contents due to elevated fluid volumes. Another possible reason that is not known to have been explored and/or utilized in PBPK modelling is the "to and fro" fluid motion across intestine which might cause these secondary peaks. ${ }^{61}$ A further possibility gastric emptying which is known to occur as a sequence of spurts. ${ }^{62}$ However, the remaining food in the stomach might not be sufficient to trigger repeated GB emptying and/or elevated hepatic secretions. This is because in vivo studies have shown no evidence of high biliary secretions in duodenal aspirates, expect from the initial high peak in [BS] due to GB emptying, after continuous or intermittent feeding with the $[\mathrm{BS}]$ equal to the fasted values on average. ${ }^{54}$ This might related to the feedback mechanism controlling GB contractions. ${ }^{63}$ The model doesn't allow the GB to contract before five gastric emptying half-life $(\sim 4 \mathrm{~h})$ have been completed. This is also in accordance with the available experimental information where the shortest time intervals between meals is $4 \mathrm{~h}$ showing repeating GB contractions without the GB reaching its maximum volume and then entering to the fasting filling-emptying cycles. It should be also pointed out that the model was able to capture the drop and rise of [BS] in fasting periods when only water is provided; see Fig.4F and individual profiles Fig4F(a'-d') between $4.5-6.5 \mathrm{~h}$.
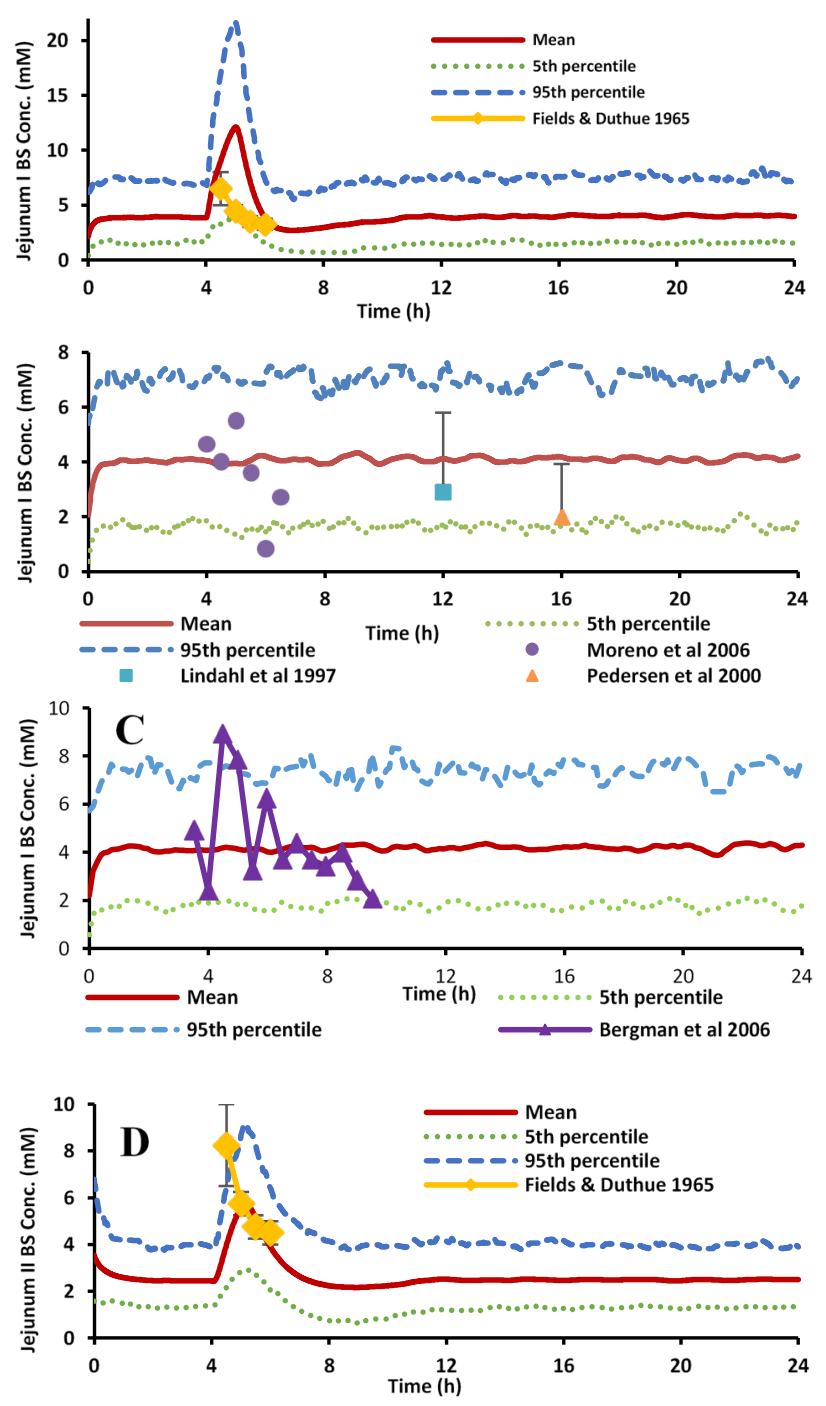

Fig.5.Time-dependent Bile Salt outputs (BS Conc. $\mathrm{mM}$ ) of ADBSM in Jejunum I-II overlaid with in vivo observations. The simulation was performed at different states according to the protocol of the clinical study: A) fed; B) fasted; C) fasted; D) fed (see supplementary material).

Fig. 5 shows the time-dependent $[\mathrm{BS}]$ in the proximal jejunum (in the ADAM model this corresponds to jejunum I, Fig.5 A - C) and distal jejunum (ADAM model jejunum II, Fig.5 D). In the Fields \& Duthue study, sampling was conducted in different segments of the small intestine. The model picks up the range in most of the observed data, including variability (see Fig. 5A, Fig. 5B and Fig. 5C). However, in the case of jejunum II (Fig. 5D), the maximum [BS] appeared sooner compared to the simulated profile. Looking at the simulated individual profiles this could be because of differences in the transit times used in the model and those of the participants in the Fields \& Duthue study. However, this information is not directly available from the in vivo study, but perhaps the transit times are more rapid compared to the values in Simcyp ${ }^{\circledR}$ library. Thus, the model doesn't capture the Tmax for this study, but this could be improved by adjusting the transit time rate constant. In this simulation only 3 virtual individuals were found to have sufficiently rapid transit times after a meal. The majority of the simulated profiles had Tmax $>$ $0.5 \mathrm{~h}$ with mean $=1.2 \pm 1.5 \mathrm{~h}$ after a meal. This reflects the physiology of the selected virtual population (Healthy Volunteers). The fact that the model can capture the observed data for most of the studies, shows that the built-in distribution of the transit times reflects actual population distribution here. The physiological parameters provided by Simcyp $\AA$, in this case fluid transit times, is based on an extensive meta-analysis covering separately different populations and different moieties, i.e. fluids, tablets, fine particles and so on. So, in this work the fluid transit times reflect BS 
transit times. However, the transit times used in publications, where a bile salt model was built to capture the time-dependent concentration profiles of BS, have been derived from mixed populations ${ }^{64}$ whereas in other models covering different aspects, e.g. bile salt metabolism, the same authors may have used different intestinal transit times. ${ }^{65}$ Looking into the literature, it was found that this is the case for most of the developed, reported EHC models ${ }^{66}$ while in some cases calibration of the model with respect to intestinal fluid transit times has been performed based on values for non-disintegrating tablets ${ }^{67}$ which are not expected to match fluid transit. ${ }^{68}$ With regards to the baseline [BS], the lowest observed [BS] was $0.83 \mathrm{mM}$ in Moreno et al (2006) compared to the predicted lowest [BS] at steady state of $1.2 \mathrm{mM}$ (fig. 5B). Based on model structure, [BS] cannot go below the baseline concentration assigned to an individual. This can be improved by either increasing $\% \mathrm{CV}$ or decreasing the mean baseline [BS] or increase the passive absorption rate (Pappcoef, Table 3). In the first case, the range of the confidence intervals will be wider, allowing the observed data to be within this range, whereas in the second and third cases the simulated curves would shift toward lower [BS]. In any case any change should be justified from meta-analysis of the available data in the public domain. Furthermore, any change to the jejunum I segment would have a knock-on effect on the time-dependent [BS] profile in remaining aboral segments. Thus further adjustment should be done to predict [BS] from other studies. However, the purpose of this work was to evaluate the predictivity of the model, without adjusting it according to the observed values of individual studies.

Fig. 6 shows the time-dependent [BS] in Ileum I (fig.6A) and Ileum IV (fig.6B-C). In the case of Ileum I, the increase in $[\mathrm{BS}]$ occurs at the same rate for the simulated and observed data and this extends to high [BS] for the simulated profile. Repeating the procedure performed for the Riethorst et al 2016 study, it was found that a virtual individual, matching the mean observed profile from Fields \& Duthue 1965 study, should have $\mathrm{Jmax}=2.83$ and $\mathrm{Km}=0.67$ (active absorption kinetics) which is higher than the mean values, $\mathrm{Jmax}=2.47 \mathrm{Km}=0.58$, as well as lower fluid volumes compared to the mean profile and shorter Tmax; mean $=6.42 \mathrm{~h}$, selected virtual individual $4.8 \mathrm{~h}$ (SF10.B). 

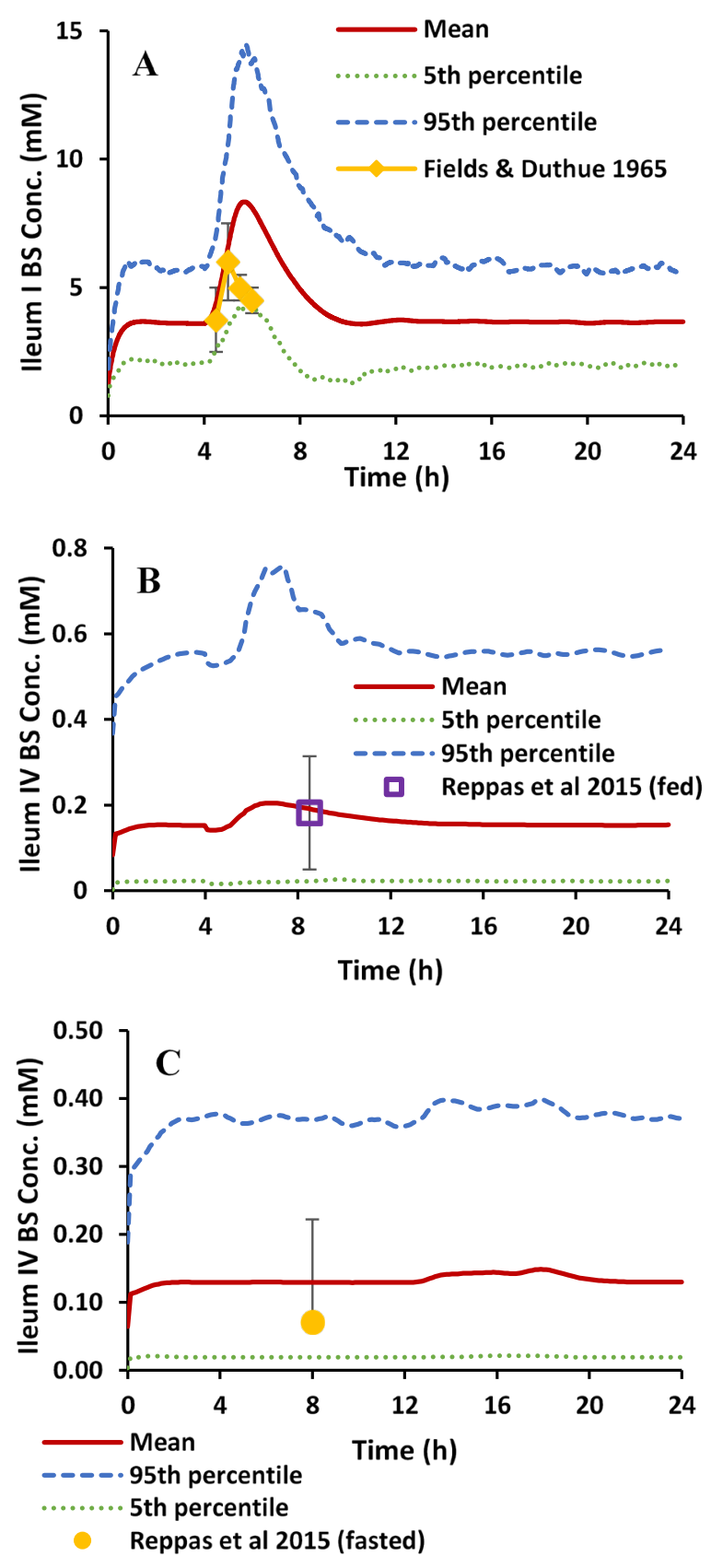

Fig.6. Mean time-dependent Bile Salt concentrations (BS Conc. mM) of ADBSM in Ileum I\&IV overlaid with in vivo observations. The simulation was performed at different states according to the protocol of the clinical study: A) fed; B) fed; C) fasted (see supplementary material).

Both absorption kinetics and transit times are not easy to appropriately-mechanistically reproduce. There are limited perfusion studies in humans for bile salt absorption in different regions of the GI tract. Absorption is more complex when a mixture of bile salts is perfused compared to a single bile salt. ${ }^{23}$ Furthermore, the available perfusion studies have been conducted for specific bile salts and hence the absorption kinetics in the current model are applied to a fraction of the total [BS], reflecting the fraction of that specific BS.

However, the composition of bile salts is not the same between populations ${ }^{2}$ and not all the studies provide this information. With respect to the transit times (residence times), used in the ADAM model these are 
derived only from scintigraphy and/or MRI studies which give the total and not the regional (segmental) transit times in the small intestine. The regional transit values used in the ADAM model assume residence time is proportional to anatomical length - population variability in regional residence times is derived from population variability in these lengths. To the authors knowledge even where regional transit has been assessed for a particular GI tract region ${ }^{69}$ there is a lack of information on total small intestinal transit time in the same subjects. However, a priori it seems reasonable that slow or fast transit times can occur within the same and/or different regions, even of the same length due to different motility patterns, e.g. forward or backward propagating waves. ${ }^{61}$ In spite of these issues, the model can capture the mean values of [BS] as reported by Reppas et al 2015 (Fig.6B). It should be mentioned that the terminal ileum is a complicated region with distinctive motility with respect to the distal ileum, which regulates emptying into the colon. However, the model captures adequately the observed data with the mean simulated [BS] profile matching surprisingly closely the observed mean [BS] (Fig.6B), taking into account that the model was informed only with the meal time and the number of subjects.

Fig.7 shows the mean simulated profile of [BS] in the colon. The observed mean [BS] is slightly lower $(\sim 0.11 \mathrm{mM})$ than the 5th percentile curve $(0.125 \mathrm{mM})$. Diakidou et al 2009 aspirated contents of the ascending colon whereas in the ADAM model at the time of writing there is a single segment for the colon. Similar adjustments, as mentioned above in the Moreno et al 2006 study (Fig.5B), could be applied to the model.

Specifically, higher passive absorption can be applied alongside higher fraction of bile salt available for absorption. However, colonic absorption is more complicated, as metabolism of the conjugated bile salts takes place by the microbiota and absorption kinetics of the unconjugated BS will in general differ from the conjugated forms. The current model uses absorption kinetics of unconjugated BS based on the available data ${ }^{24}$ but it does not account for luminal metabolism. Furthermore, the passive absorption coefficient is derived from a single bile acid, specifically chenodeoxycholate (CDCA). However, this parameter is expected to differ for each bile acid albeit such information is not available. Furthermore, the mean fluid volume in the colonic compartment was $\sim 10 \mathrm{~mL}$ whereas Diakidou et al 2009 reported volumes of 22.3 $\pm 7.7 \mathrm{~mL}$. This is double the baseline fluid volumes in the ADAM model and higher volumes would bring

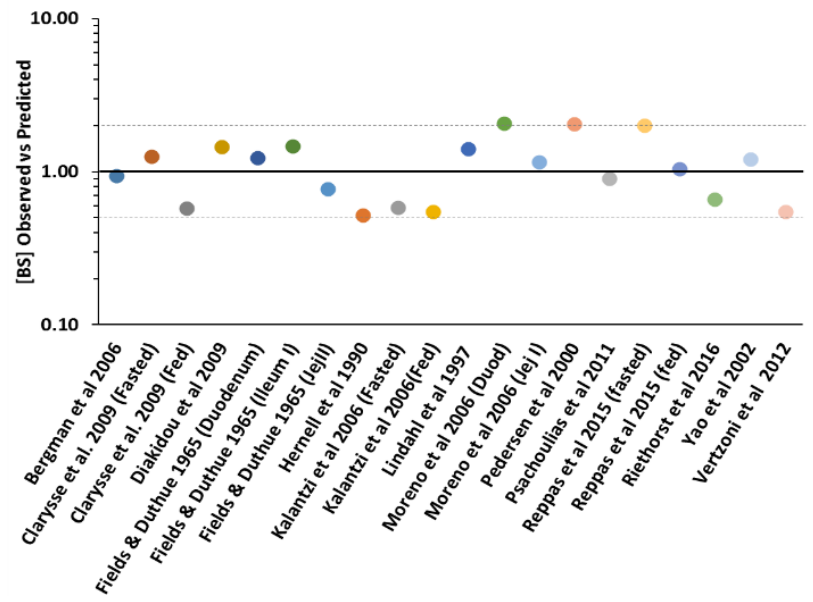

[BS] down to the observed values. Indeed, changing the default values of the fluid volumes (mean=11.92 $\mathrm{mL}, \% \mathrm{CV}=90$ ), to mean $=22.3 \mathrm{~mL}$ and $\% \mathrm{CV}=34.5$, the mean simulated $[\mathrm{BS}]$ is much closer to the observed data (SF11B).

Fig.7. Mean time-dependent Bile Salt concentrations (BS Conc. mM) of ADBSM in colon overlaid with in vivo observations. The simulation was performed at fasted state according to the protocol of the clinical study (see supplementary material). 
Fig. 8 shows the overall predictiveness of the model by taking the ratio of observed to predicted [BS] values. The ratios have been calculated from the mean of the all-time-dependent observed data divided by the predicted values within the same time period. All the ratios were within two fold with most of them

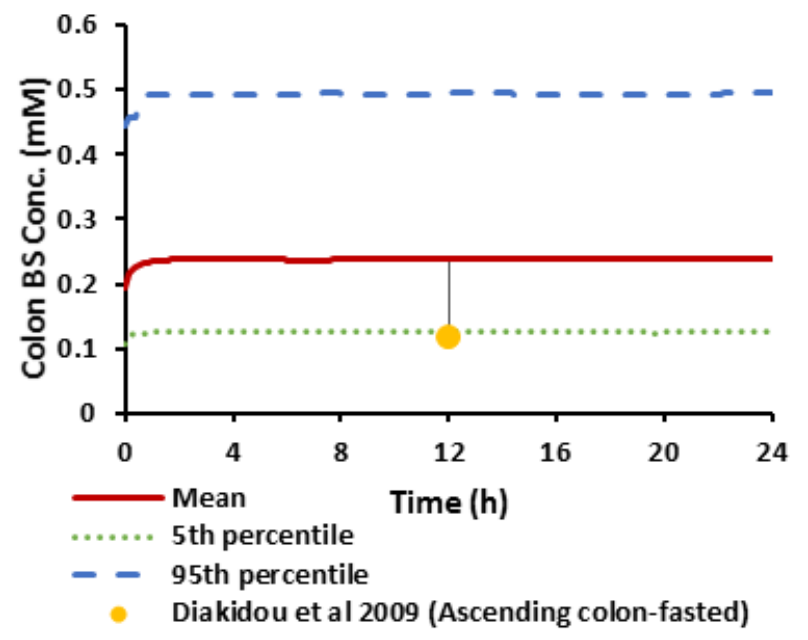

$(11 / 20)$ within 1.25 fold.

Fig.8. Observed versus predicted [BS $\left.\mathrm{BStal}_{\mathrm{ta}}\right]$ ratio. The solid line represents the line of unity and the dotted lines the 2-fold error thresholds.

\section{CONCLUSIONS}

A population-based PBPK model was developed for the prediction of total bile salt disposition within the GI luminal fluids. The available studies for performance verification of the model provided only the meal time, the number of the subjects and their gender and age. The model was not adjusted or optimized to better simulate a specific study. The development of the model was based on current knowledge of human physiology and its predictivity was assessed using in vivo studies providing only luminal bile salt concentrations. The time variant luminal [BS] is a complex interplay of intestinal transit times, fluid volumes, absorption, secretions and the time course of a low or high fat meal with or without water intake at different times. Also, the sampling technique and intensity of the sampling can interrupt the EHC of BS to various extents, resulting in alterations to luminal [BS], adding more complexity to accurate prediction of [BS] over time. However, it is essential to have deep knowledge of human physiology and the drawbacks/limitations of the methods used to obtain these data. It is the intention of this work to further contribute to the overall effort for more mechanistic PBPK models enabling improved predictivity of oral absorption.

\section{AUTHOR INFORMATION}

\section{Corresponding Author}

*Konstantinos.Stamatopoulos@certara.com, stamato_k@hotmail.com

Konstantinos Stamatopoulos 


\title{
Author Contributions
}

${ }^{\dagger} \mathrm{K} . \mathrm{S}$ conducted literature review, meta-analysis, developed the Advanced Dynamic Bile Salt Model (ADBSM) and wrote the manuscript, S.M.P and D.T supervised and approved the research work and overviewed manuscript preparation. K.S, S.M.P and D.T reviewed and updated dynamic water volumes model. L.M provided in vivo data for the human Gallbladder and overviewed manuscript preparation.

\section{Acknowledgments}

Funding: This work was supported by FDA Grant (No. 1U01FD005865) and the Simcyp Consortium.

The views expressed in this article are those of the authors and should not be construed to represent the U.S. Food and Drug Administration (FDA)'s views or policies. The mention of commercial products, their sources, or their use in connection with material reported herein is not to be construed as either an actual or implied endorsement of such products by the FDA.

\begin{abstract}
ABBREVIATIONS
ADBSM, advanced dynamic bile salt model; BS, bile salts; [BS], bile salt concentration; GB, gallbladder; GBV, gallbladder volume; mGBV, maximum gallbladder volume; IMMC, intermediate migrating motor complex; CDCA chenodeoxycholate; GCDC, glycochenodeoxycholic acid; EHC, enterohepatic circulation; CDCA, chenodeoxycholate; KAbs, the absorption rate constant of bile salts; BSm, the bile salt mass $(\mathrm{mmol}) ; \mathrm{kt}$, transit rate constant $\left(\mathrm{h}^{-1}\right) ;$ BSConc, time-dependent [BS] (mM); BSConc baseline $_{\text {, }}$ [BS] baseline

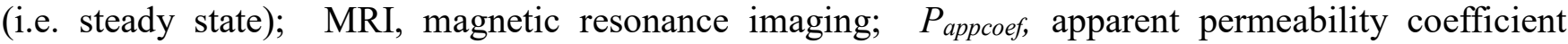
$(\mathrm{mmol} / \mathrm{h} / \mathrm{mM})$; Jmax, maximum rate of bile acid uptake $(\mathrm{mmol} / \mathrm{h}) ; \mathrm{Km}$, Michaelis-Menten constant (mmol); PV, performance verification; emptying rate constant $k_{\text {GBemfed }}$, emptying rate constant $\left(\mathrm{h}^{-1}\right)$; $c_{\text {filling, }}$, refilling rate $(\mathrm{mL} / \mathrm{h})$; Hsr, Hepatic bile secretions; $H B S_{\text {massrate }}$, hepatic bile salt mass rate $(\mathrm{mmol} / \mathrm{h}) ; \mathrm{Vi}(\mathrm{t})$, time-dependent fluid volume in the fasted or fed state in the $i^{\text {th }}$ ADAM GI tract segment; $B S m$, bile salt mass (mmol) in each segment;
\end{abstract}

\section{Supporting Information}

Trial designs of the clinical studies used for model verification, outputs of ADBSM and all ADAM GI compartments for each clinical study.

\section{REFERENCES}

1. van Berge Henegouwen, G. P.; Hofmann, A. F. Nocturnal gallbladder storage and emptying in gallstone patients and healthy subjeGastroenterologł978, 75, (5), 87985.

2. Riethorst, D.; Mols, R.; Duchateau, G.; Tack, J.; Brouwers, J.; Augustijns, P. Characterization of Human Duodenal Fluids in Fasted and Fed State Conditionsnal of Pharmaceutical Sciences 2016, $105,(2), 673681$.

3. Luiking, Y. C.; Kloppers, N. J.; Reofs, J. M.; Nieuwenhuijs, V. B.; Peeters, T. L.; Akkermans, L. M.; van Berge Henegouwen, G. P. Effects of intraduodenal bile on interdigestive gastrointestinal and gallbladder motility in healthy subjedigestion2001,63, (3), 195202.

4. Di Ciaula,A.; Covelli, M.; Berardino, M.; Wang, D. Q.; Lapadula, G.; Palasciano, G.; Portincasa, $P$. Gastrointestinal symptoms and motility disorders in patients with systemic scleroßetQha. GastroenterologR2008,8, (1), 7. 
5. Keszthelyi, D.; Knol, D.; Troost, F. J.; van Avesaat, M.; Foltz, M.; Masclee, A. A. Time of ingestion relative to meal intake determines gastrointestinal responses to a plant sterol-containing yoghurt drink.European journal of nutritio2013,52, (4), 1417-20.

6. Portincasa, P.; Moschetta, A.; Colecchia, A.; Festi, D.; Palasciano, G. Measurements of gallbladder motor function by ultrasonography: towards standardizæligastive and Liver Disease2003,35, 56-61.

7. Portincasa, P.; Di Ciaula, A.; Bal G. Gallbladder motor function in gallstone patients: sonographic and in vitro studies on the role of gallstones, smooth muscle function and gallbladder wall inflammatiournal of hepatology 1994,21, (3), 43040.

8. Chan, D. C.; Chang, T. M.; Chen, C. J.; Chen, T. W.; Yu, J. C.; Liu, Y. C. Gallbladder contractility and volume characteristics in gallstone dyspepbicald journal of gastroenterology 2004, 10, (5), 7214.

9. Portincasa, P.; Olschetta, A.; Berardino, M.; Eliaula, A.; Vacca, M.; Baldassarre, G.; Pietrapertosa, A.; Cammarota, R.; Tannoia, N.; Palasciano, G. Impaired gallbladder motility and delayed orocecal transit contribute to pigment gallstone and biliary sludge formalbietai thalassemia major adultkKorld journal of gastroenterolog3004, 10, (16), 238390.

10. Portincasa, P.; Peeters, T. L.; van Betrbenegouwen, G. P.; van Solinge, W. W.; Palasciano, G.; van Erpecum, K. J. Acute intraduodenal bile salt depletiøs leastrong gallbladder contraction, altered antroduodenal motility and high plasma motilin levels in humłzerogastroenterology and motility : the official journal of the European Gastrointestinal Motility So@i00, 12, (5), 421-30.

11. Grøndahl,M. F.; Bagger, J. I.; Lund, A.; Faurschou, A.; Rehfeld, J. F.; Holst, J. J.; Vilsbøll, T.; Knop, F. K. Effects of Smoking Versus Nonsmoking on Postprandial Glucose Metabolism in Heavy Smokers Compared With Nonsmokerabetes Care018,41, (6), 12601267.

12. Marzio, L.; Falcucci, M.; Ciccaglione, A. F.; Malatesta, M. G.; Lapenna, D.; Ballone, E.; Antonelli, C.; Grossi, L. Relationship between gastric and gallbladder emptying and refilling in normal subjects and patients with $\mathrm{H}$. pyłpoisitive and-negative idiopathic dyspepsia and correlation with symptomsigestive diseases and scien\$996,41, (1), 2631.

13. Stolk, M. F.; van Erpecum, K. J.; Smout, A. J.; Akkermans, L. M.; Jansen, J. B.; Lamers, C. B.; Peeters, T. L.; vanBergdenegouwen, G. PMotor cycles with phase III in antrum are associated with high motilin levels and prolonged gallbladder emptyingge American journal of physiology 1993,264, (4 Pt 1), G596600.

14. Gourtsoyiannis, N. C.; Damilakis, J. E.; Charoulakis, N. Z.; BakanAal\$; Vlahonikolis, J. G.; Xynos, E. Relationship of gallbladder contour, fasting volume and emptying to body size indices in normal subjects and patients with gallstorigestion1995,56, (5), 3959.

15. Palasciano, G.; Serio, G.; Portincasa, P.mPerl, V.; Fanelli, M.; Velardi, A.; Calo' Gabrieli, B.; Vinciguerra, V. Gallbladder volume in adults, and relationship to age, sex, body mass index, and gallstones: a sonographic population stubye American journal of gastroenterolo\$\$92,87, (4), 493-7.

16. Marciani, L.; Hall, N.; Pritchard, S. E.; Cox, E. F.; Totman, J. J.; Lad, M.; Hoad, C. L.; Foster, T. J.; Gowland, P. A.; Spiller, R. C. Preventing Gastric Sieving by Blending a Solid/Water Meal Enhances Satiation in Healthy Huma灭e Journabf Nutrition2012, 142 (7), 12531258.

17. Hussein, M. O.; Hoad, C. L.; Wright, J.; Singh, G.; Stephenson, M. C.; Cox, E. F.; Placidi, E.; Pritchard, S. E.; Costigan, C.; Ribeiro, H.; Ciampi, E.; Nandi, A.; Hedges, N.; Sanderson, P.; Peters, H. P.; Rayme,rP.; Spiller, R. C.; Gowland, P. A.; Marciani, L. Fat Emulsion Intragastric Stability and Droplet Size Modulate Gastrointestinal Responses and Subsequent Food Intake in Young Adults. The Journal of Nutritioß2015, 145, (6), 11701177.

18. Marciani, L.;Wickham, M.; Singh, G.; Bush, D.; Pick, B.; Cox, E.; Fillerryavis, A.; Faulks, R.; Marsden, C.; Gowland, P. A.; Spiller, R. C. Enhancement of intragastric acid stability of a fat 
emulsion meal delays gastric emptying and increases cholecystokinin release and gallbladder contraction American journal of physiology. Gastrointestinal and liver physioRog7,292 (6), G160713.

19. Marciani, L.; Cox, E. F.; Hoad, C. L.; Totman, J. J.; Costigan, C.; Singh, G.; Shepherd, V.; Chalkley, L.; Robinson, M.; IsorrR.; Gowland, P. A.; Spiller, R. C. Effects of various food ingredients on gall bladder emptyintguropean Journal of Clinical Nutritio2013,67, (11), 11821187.

20. Dooley, C. P.; Di Lorenzo, C.; Valenzuela, J. E. Variability of migrating motor complex in humans Digestive diseases and scien\$992,37, (5), 723728.

21. Luiking, Y. C.; van der Reijden, A. C.; van Berge Henegouwen, G. P.; Akkermans, L. M. Migrating motor complex cycle duration is determined by gastric or duodenal origin of phase III. The American journal of physiolog998,275, (6 Pt 1), G124651.

22. Marzio, L.; Neri, M.; Capone, F.; Di Felice, F.; De Angelis, C.; Mezzetti, A.; Cuccurullo, F. Gallbladder contraction and its relationship to interdigestive duodenal motor activity in normal human subjectsDigestive diseases and scien $₫ 988,33,(5), 5404$.

23. Krag, E.; Phillips, S. F. Active and passive bile acid absorption in man. Perfusdiersstfthe ileum and jejunumThe Journal of clinical investigatio1974,53, (6), 16861694.

24. Mekhjian, H. S.; Phillips, S. F.; Hofmann, A. F. Colonic absorption of unconjugated bile acids: perfusion studies in maD.igestive diseases and scien\$979,24, (7), 54550.

25. Birru, W. A.; Warren, D. B.; Ibrahim, A.; Williams, H. D.; Benameur, H.; Porter, C. J. H.; Chalmers, D. K.; Pouton, C. W. Digestion of Phospholipids after Secretion of Bile into the Duodenum Changes the Phase Behavior of Bile Compts Molecular pharmaceutic 3014,11 , (8), 28252834.

26. Mudie, D. M.; Amidon, G. L.; Amidon, G. E. Physiological Parameters for Oral Delivery and in Vitro Testing.Molecular pharmaceutic\$010, 7, (5), 13881405.

27. Perez de la Cruz Moreno, M.; Otthl.; Deferme, S.; Lammert, F.; Tack, J.; Dressman, J.; Augustijns, P. Characterization of faste human intestinal fluids collected from duodenum and jejunum.The Journal of pharmacy and pharmacolQg,06,58, (8), 107989.

28. Lindahl, A.; Ungell,A. L.; Knutson, L.; Lennernas, H. Characterization of fluids from the stomach and proximal jejunum in men and womfernarmaceutical researck997, 14, (4), 497 502.

29. Pedersen, B. L.; Mullertz, A.; Brondsted, H.; Kristensen, H. G. A comparison ofltheilisy of danazol in human and simulated gastrointestinal fluptessmaceutical researc2000,17, (7), $891-4$.

30. Deferme, S.; Tack, J.; Lammert, F.; Augustijns, PglyPeoprotein attenuating effect of human intestinal fluid. Pharmaceutical researc2003,20, (6), 9003.

31. Tangerman, A.; van Schaik, A.; van der Hoek, E. W. Analysis of conjugated and unconjugated bile acids in serum and jejunal fluid of normal subjectsinica Chimica Acta986, 159, (2), 123 132.

32. Vertzoni, M.; Goumas, K.; Soderlind, E.; Abrahamsson, B.; Dressman, J. B.; Poulou, A.; Reppas, C. Characterization of the ascending colon fluids in ulcerative cofltisrmaceutical research 2010,27, (8), 16206.

33. Soderlind, E.; Karlsson, E.; CarlssoA.; Kong, R.; Lenz, A.; Lindborg, S.; Sheng, J. J. Simulating fasted human intestinal fluids: understanding the roles of lecithin and bile acids. Molecular pharmaceutic8010, 7, (5), 1498507.

34. Pathak, S. M.; Ruff, A.; Kostewicz, E. S.; Patel, Nurner, D. B.; Jamei, M. Modeßased Analysis of Biopharmaceutic Experiments To Improve Mechanistic Oral Absorption Modeling: An Integrated in Vitro in Vivo Extrapolation Perspective Using Ketoconazole as a Model Drug. Molecular pharmaceutic8017, 14, (12), 43054320. 
35. Parrott, N.; Lukacova, V.; Fraczkiewicz, G.; Bolger, M. B. Predicting Pharmacokinetics of Drugs Using Physiologically Based Modeling-Application to Food Effects. The AAPS Journ2009, 11, (1), 4553.

36. Peeters, T. L.; Vantrappen, Ganssens, J. Bile acid output and the interdigestive migrating motor complex in normals and in cholecystectomy pati@奴oenterologł980,79, (4), 678 81.

37. Shaffer, E. A. Review article: control of g(dlladder motor functionAlimentary pharmaology \& therapeutic\$2000, 14 Suppl 22-8.

38. Mudie, D. M.; Murray, K.; Hoad, C. L.; Pritchard, S. E.; Garnett, M. C.; Amidon, G. L.; Gowland, P. A.; Spiller, R. C.; Amidon, G. E.; Marciani, L. Quantification of gastrointestinal liquid volumes and disibution following a $240 \mathrm{~mL}$ dose of water in the fasted stededecular pharmaceutic $8014,11,(9), 303947$.

39. Khalaf, A.; Hoad, C. L.; Menys, A.; Nowak, A.; Taylor, S. A.; Paparo, S.; Lingaya, M.; Falcone, Y.; Singh, G.; Spiller, R. C.; Gowland, P. A.; Marciani, L.; Moran, G. W. MRI assessment of the postprandial gastrointestinal motility and peptide responbeaithy humans.

Neurogastroenterology and motility : the official journal of the European Gastrointestinal Motility Society2018,30, (1).

40. Sonne, D. P.; Rehfeld, J. F.; Holst, J. J.; Vilsboll, T.; Knop, F. K. Postprandial gallbladder emptying in patiats with type 2 diabetes: potential implications for torilduced secretion of glucagonlike peptide 1 European journal of endocrinolog014, 171, (4), 40719.

41. Guiastrennec, B.; Sonne, D. P.; Hansen, M.; Bagger, J. I.; Lund, A.; Rehfeld, J. Fr; $\mathbb{Q l s ̧ k a ~}$ Karlsson, M. O.; Vilsboll, T.; Knop, F. K.; Bergstrand, M. Mechanßased Modeling of Gastric Emptying Rate and Gallbladder Emptying in Response to Caloric Iotake. pharmacometrics \& systems pharmacolagk6,5, (12), 692700.

42. Abbiati, R.A.; Manca, D. Enterohepatic Circulation Effect in Physiologically Based Pharmacokinetic Models: The Sorafenib Calseustrial \& Engineering Chemistry Research 2017,56, (12), 31563166.

43. Jain, L.; Woo, S.; Gardner, E. R.; Dahut, W. L.; Kohn, E. Gimknar, S.; Mould, D. R.; Giaccone, G.; Yarchoan, R.; Venitz, J.; Figg, W. D. Population pharmacokinetic analysis of sorafenib in patients with solid tumoußsitish journal of clinical pharmacolog\$011, 72, (2), 294305.

44. Lehr, T.; Staab, A.; Tillmam, C.; Trommeshauser, D.; Schaefer, H. G.; Kloft, C. A quantitative enterohepatic circulation model: development and evaluation with tesofensine and meloxicam. Clinical pharmacokinetic $2009,48,(8), 52942$.

45. Jazrawi, R. P. Review article: measuretrofrgall-bladder motor function in health and disease. Alimentary pharmacology \& therapeut/2000,14 Suppl 227-31.

46. Torsoli, A.; Corazziari, E.; Habib, F. I.; Cicala, M. Pressure relationships within the human bile tract. Normal and abnormal phøsigy. Scandinavian journal of gastroenterology. Supplement 1990, 175, 52-7.

47. Kher, K.; Guelrud, M. Normal sphincter of oddi motor functi@osrent gastroenterology reports2004,6, (2), 1638.

48. Pallotta, N.; Corazziari, E.; Scopinaro, F.; BoniRa, Schillaci, O.; Vignoni, A.; Mangano, M. ... And Dangers of Clinging to Old Theories Based on Inconsistent Daterican Journal Of Gastroenterologł999,94, 1705.

49. Kellow, J. E.; Borody, T. J.; Phillips, S. F.; Tucker, R. L.; Haddad, A. C. Humterdigestive motility: variations in patterns from esophagus to coleastroenterologł986,91, (2), 38695.

50. Nilsson, B. I.; Svenberg, T.; Tollstrom, T.; Hellstrom, P. M.; Samuelson, K.; Schnell, P. O. Relationship between interdigestive gallbladeløptying, plasma motilin and migrating motor complex in manActa physiologica Scandinaviđ\$90, 139, (1), 5561. 
51. Toouli, J.; Bushell, M.; Stevenson, G.; Dent, J.; Wycherley, A.; Iannos, J. Gallbladder emptying in man related to fasting duodenalgmiting motor contractions.he Australian and New Zealand journal of surgery1986,56, (2), 14751.

52. Howard, P. J.; Murphy, G. M.; Dowling, R. H. Gall bladder emptying patterns in response to a normal meal in healthy subjects and patients with gatleat: ultrasound stud\&ut1991,32, (11), 14061411.

53. Cano, N.; Cicero, F.; Ranieri, F.; Martin, J.; di Costanzo, J. Ultrasonographic study of gallbladder motility during total parenteral nutritionGastroenterologł986,91, (2), 3137.

54. Mok, H. Y.; von Bergmann, K.; Grundy, S. M. Effects of continuous and intermittent feeding on biliary lipid outputs in man: application for measurements of intestinal absorption of cholesterol and bile acidsJournal of lipid research979,20, (3), 38998.

55. Northfield, T. C.; Hofmann, A. F. Biliary lipid output during three meals and an overnight fast. I. Relationship to bile acid pool size and cholesterol saturation of bile in gallstone and control subjects.Gut1975, 16, (1), 111.

56. Yao, L.; Heubi, J. E.Buckley, D. D.; Fierra, H.; Setchell, K. D.; Granholm, N. A.; Tso, P.; Hui, D. Y.; Woollett, L. A. Separation of micelles and vesicles within lumenal aspirates from healthy humans: solubilization of cholesterol after a meaurnal of lipid researc2002, 43, (4), 65460.

57. Kalantzi, L.; Goumas, K.; Kalioras, V.; Abrahamsson, B.; Dressman, J. B.; Reppas, C. Characterization of the human upper gastrointestinal contents under conditions simulating bioavailability/bioequivalence studießharmaceutical researc2006,23, (1), 16576.

58. ØstefJØrgensen, E.; Qvist, N.; Pedersen, S. A.; Rasmussen, L.; Hovendal, C. P. Postprandial Gallbladder Emptying Is Related to Intestinal Motility at the Time of Meal Ingestion. Scandinavian journal ofagstroenterology992,27, (8), 699702.

59. Dowling, R. H.; Mack, E.; Small, D. M. Effects of controlled interruption of the enterohepatic circulation of bile salts by biliary diversion and by ileal resection on bile salt secretion, synthesis, and pool size in the rhesus monk 区xe Journal of clircal investigation1970,49, (2), 23242.

60. Jazrawi, R. P.; Pazzi, P.; Petroni, M. L.; Prandini, N.; Paul, C.; Adam, J. A.; Gullini, S.; Northfield, T. C. Postprandial gallbladder motor function: refilling and turnover of bile in health and in cholelitirasis. Gastroenterologł995, 109, (2), 58291.

61. Yu, A.; Jackson, T.; Tsume, Y.; Koenigsknecht, M.; Wysocki, J.; Marciani, L.; Amidon, G. L.; Frances, A.; Baker, J. R.; Hasler, W.; Wen, B.; Pai, A.; Sun, D. Mechanistic Fluid Transport Model to Estimat@astrointestinal Fluid Volume and Its Dynamic Change Over The. $A A P S$ Journal2017, 19, (6), 16821690.

62. Yokrattanasak, J.; De Gaetano, A.; Panunzi, S.; Satiracoo, P.; Lawton, W. M.; Lenbury, Y. A Simple, Realistic Stochastic Model of Gastric Enipgyy PLOS ONE2016, 11, (4), e0153297.

63. van Ooteghem, N. A. M.; Moschetta, A.; Rehfeld, J. F.; Samsom, M.; van Erpecum, K. J.; van BergeHenegouwen, G. P. Intraduodenal conjugated bile salts exert negative feedback control on gall bladder emptying inte fasting state without affecting cholecystokinin release or antroduodenal motilityGut2002,50, (5), 669674.

64. Hofmann, A. F.; Molino, G.; Milanese, M.; Belforte, G. Description and simulation of a physiological pharmacokinetic model for the metwo and enterohepatic circulation of bile acids in man. Cholic acid in healthy marhe Journal of clinical investigatio1983,71, (4), 100322.

65. Hofmann, A. F.; Cravetto, C.; Molino, G.; Belforte, G.; Bona, B. Simulation of the metabolism and enterbepatic circulation of endogenous deoxycholic acid in humans using a physiologic pharmacokinetic model for bile acid metabolis@astroenterologł987,93, (4), 693709.

66. Zuo, P.; Dobbins, R. L.; O'Conn\$emmes, R. L.; Young, M. A. A Systems Model for Ursodeoxycholic Acid Metabolism in Healthy and Patients With Primary Biliary Cirrh6\$\$: pharmacometrics \& systems pharmacolegr6,5, (8), 41826. 
67. Sips, F. L. P.; Eggink, H. M.; Hilbers, P. A. J.; Soeters, M. R.; Groen, A. K.; van Riel, N. A. W. In Silico Analysis Identifies Intestinal Transit as a Key Determinant of Systemic Bile Acid Metabolism.Front Physiol2018,9, 631-631.

68. Davis, S. S.; Hardy, J. G.; Fara, J. W. Transit of pharmaceutical dosage forms through the small intestine.Gut1986,27, (8), 88692.

69. Quon, M. G.; Mena, I.; Valenzuela, J. E. Abnormalities in the duodenal transit and motility in duodenal ulcer patients: studies with a new isotopic techn@eate.989,30, (5), 579585.

70. Vertzoni, M.; Markopoulos, C.; Symiddes, M.; Goumas, C.; Imanidis, G.; Reppas, C. Luminal Lipid Phases after Administration of a Triglyceride Solution of Danazol in the Fed State and Their Contribution to the Flux of Danazol Across Ce\&Cell MonolayersMolecular pharmaceutics 2012,9, (5), 11891198.

71. Bergman, E.; Forsell, P.; Tevell, A.; Persson, E. M.; Hedeland, M.; Bondesson, U.; Knutson, L.; Lennernas, $\mathrm{H}$. Biliary secretion of rosuvastatin and bile acids in humans during the absorption phase European journal of pharmaceutical ecices : official journal of the European Federation for Pharmaceutical Science日06,29, (3-4), 20514.

72. Clarysse, S.; Tack, J.; Lammert, F.; Duchateau, G.; Reppas, C.; Augustijns, P. Postprandial evolution in composition and characteristics of hurdaadenal fluids in different nutritional states Journal of Pharmaceutical Scienc\$09,98, (3), 11771192.

73. Diakidou, A.; Vertzoni, M.; Goumas, K.; Söderlind, E.; Abrahamsson, B.; Dressman, J.; Reppas, C. Characterization of the Contents of AscegdDolon to Which Drugs are Exposed After Oral Administration to Healthy Adults?harmaceutical researc2009,26, (9), 21412151.

74. Fields, M.; Duthie, H. L. Effect of vagotomy on intraluminal digestion of fat in neart.1965,6, (3), 301310.

75. Hernell, O.; Staggers, J. E.; Carey, M. C. Physcibalmical behavior of dietary and biliary lipids during intestinal digestion and absorption. 2. Phase analysis and aggregation states of luminal lipids during duodenal fat digestion in healthy adult humbeings.Biochemistry1990,29, (8), 204156.

76. Psachoulias, D.; Vertzoni, M.; Goumas, K.; Kalioras, V.; Beato, S.; Butler, J.; Reppas, C. Precipitation in and supersaturation of contents of the upper small intestine after administration of two weak bass to fasted adult\$.harmaceutical researc2011,28, (12), 314558.

77. Reppas, C.; Karatza, E.; Goumas, C.; Markopoulos, C.; Vertzoni, M. Characterization of Contents of Distal Ileum and Cecum to Which Drugs/Drug Products are Exposed During Bioavailablity/Bioequivalence Studies in Healthy Adult3harmaceutical researc2015,32, (10), 33383349. 
Table S1. Details of the clinical studies used for the Performance Verification of the ADBSM.

\begin{tabular}{|c|c|c|c|c|c|c|c|}
\hline \multirow[t]{2}{*}{ Status } & \multicolumn{2}{|c|}{ Calories } & \multirow{2}{*}{$\begin{array}{l}\text { Volume } \\
\qquad(\mathrm{mL})\end{array}$} & \multirow{2}{*}{$\begin{array}{c}\text { Time of } \\
\text { sampling }\end{array}$} & \multicolumn{2}{|c|}{ Region } & \multirow[t]{2}{*}{ Ref. } \\
\hline & Total & $\begin{array}{l}\text { Fat } \\
(\%)\end{array}$ & & & In vivo & ADAM & \\
\hline Fasting-fed & 574.4 & 26 & $\begin{array}{c}250 \\
\text { (fasting) } \\
300 \mathrm{~mL} \\
\text { liquid } \\
\text { meal+350 } \\
\text { mL water } \\
\text { (Fed) }\end{array}$ & $\begin{array}{l}\text { Immediately } \\
\text { after meal }\end{array}$ & Duodenum & Duodenum & $\begin{array}{c}\text { Clarysse et al } \\
2009\end{array}$ \\
\hline Fasting & & & - & & $\begin{array}{l}\text { Proximal } \\
\text { Jejunum }\end{array}$ & Jejunum 1 & $\begin{array}{c}\text { Pedersen et } \\
\text { al } 2000\end{array}$ \\
\hline Fasting & & & & & $\begin{array}{l}\text { Proximal } \\
\text { Jejunum }\end{array}$ & Jejunum 1 & $\begin{array}{c}\text { Moreno et al } \\
2006 \\
\end{array}$ \\
\hline \multirow[t]{2}{*}{ Fed } & \multirow[t]{2}{*}{427.7} & \multirow[t]{2}{*}{48} & \multirow[t]{2}{*}{ - } & \multirow{2}{*}{$\begin{array}{c}0.5 \mathrm{~h} \text { after } \\
\text { meal }\end{array}$} & $\begin{array}{l}\text { Proximal } \\
\text { jejunum }\end{array}$ & Jejunum 1 & \multirow{2}{*}{$\begin{array}{c}\text { Fields and } \\
\text { Duthie } 1965\end{array}$} \\
\hline & & & & & Distal jejunum & Jejunum 2 & \\
\hline Fed & & & & $\begin{array}{c}0.5 \mathrm{~h} \text { after } \\
\text { meal }\end{array}$ & Proximal ileum & Ileum I & $\begin{array}{c}\text { Fields and } \\
\text { Duthie } 1965\end{array}$ \\
\hline Fasting-fed & \multirow[t]{2}{*}{960} & \multirow[t]{2}{*}{58} & $\begin{array}{c}\text { Total } 900 \\
\mathrm{~mL} \\
\text { (fasting) }^{* *}\end{array}$ & & Distal Ileum & Ileum IV & $\begin{array}{c}\text { Reppas et al } \\
2015\end{array}$ \\
\hline Fasting-fed & & & $\begin{array}{l}\text { Total } 750 \\
\mathrm{~mL}(\text { fed) }\end{array}$ & & Caecum & Colon & $\begin{array}{c}\text { Reppas et al } \\
2015 \\
\end{array}$ \\
\hline Fasting-fed & $\begin{array}{c}960 \\
\text { (breakfast) }\end{array}$ & & $\begin{array}{c}227 \mathrm{~mL} \\
\text { (milk with } \\
\text { breakfast) } \\
250 \mathrm{~mL} \\
\text { (fruit juice) }\end{array}$ & & Ascending & Colon & $\begin{array}{l}\text { Diakidou et } \\
\text { al } 2009\end{array}$ \\
\hline Fasting-fed & 379.6 & 79.5 & $\begin{array}{l}\text { Liquid meal } \\
240 \mathrm{~mL}\end{array}$ & $\begin{array}{c}15 \text { min after } \\
\text { meal }\end{array}$ & Duodenum & Duodenum & $\begin{array}{c}\text { Yao et al } \\
2002\end{array}$ \\
\hline fed & 682.44 & 66 & $\begin{array}{l}\text { Liquid meal } \\
\quad 400 \mathrm{~mL}\end{array}$ & $\begin{array}{c}60 \text { and } 90 \mathrm{~min} \\
\text { following } \\
\text { the meal }\end{array}$ & Duodenum & Duodenum & $\begin{array}{c}\text { Hernell et al } \\
1990\end{array}$ \\
\hline Fasting-fed & 750 & 26 & $\begin{array}{l}500 \mathrm{~mL} \\
\text { liquid meal } \\
\text { (Ensure } \\
\text { Plus) }\end{array}$ & $\begin{array}{c}30,60,120 \\
\text { and } 180 \mathrm{~min} \\
\text { after meal }\end{array}$ & $\begin{array}{c}\text { Distal } \\
\text { duodenum }\end{array}$ & Duodenum & $\begin{array}{c}\text { Kalantzi et al } \\
2006\end{array}$ \\
\hline Fasting-fed & 600 & 27 & $\begin{array}{l}400 \mathrm{~mL} \\
\text { liquid meal } \\
\text { (Ensure } \\
\text { Plus) }\end{array}$ & $\begin{array}{l}30 \text { min after } \\
\text { the meal and } \\
\text { for every } 10 \\
\text { min for } 90 \mathrm{~min}\end{array}$ & Duodenum & Duodenum & $\begin{array}{l}\text { Riethorst et } \\
\text { al } 2016\end{array}$ \\
\hline
\end{tabular}

* Midintestine section 150-190cm taken as distal jejunum according to divisions determined from ICRP; ** different volumes of water were given at different time points before aspiration (see Reppas et al 2015) 
SF1. ADBSM outputs using Clarysse et al 2009 fasting-fed protocol. Blue arrow indicates water administration and red arrow the time of meal administration.
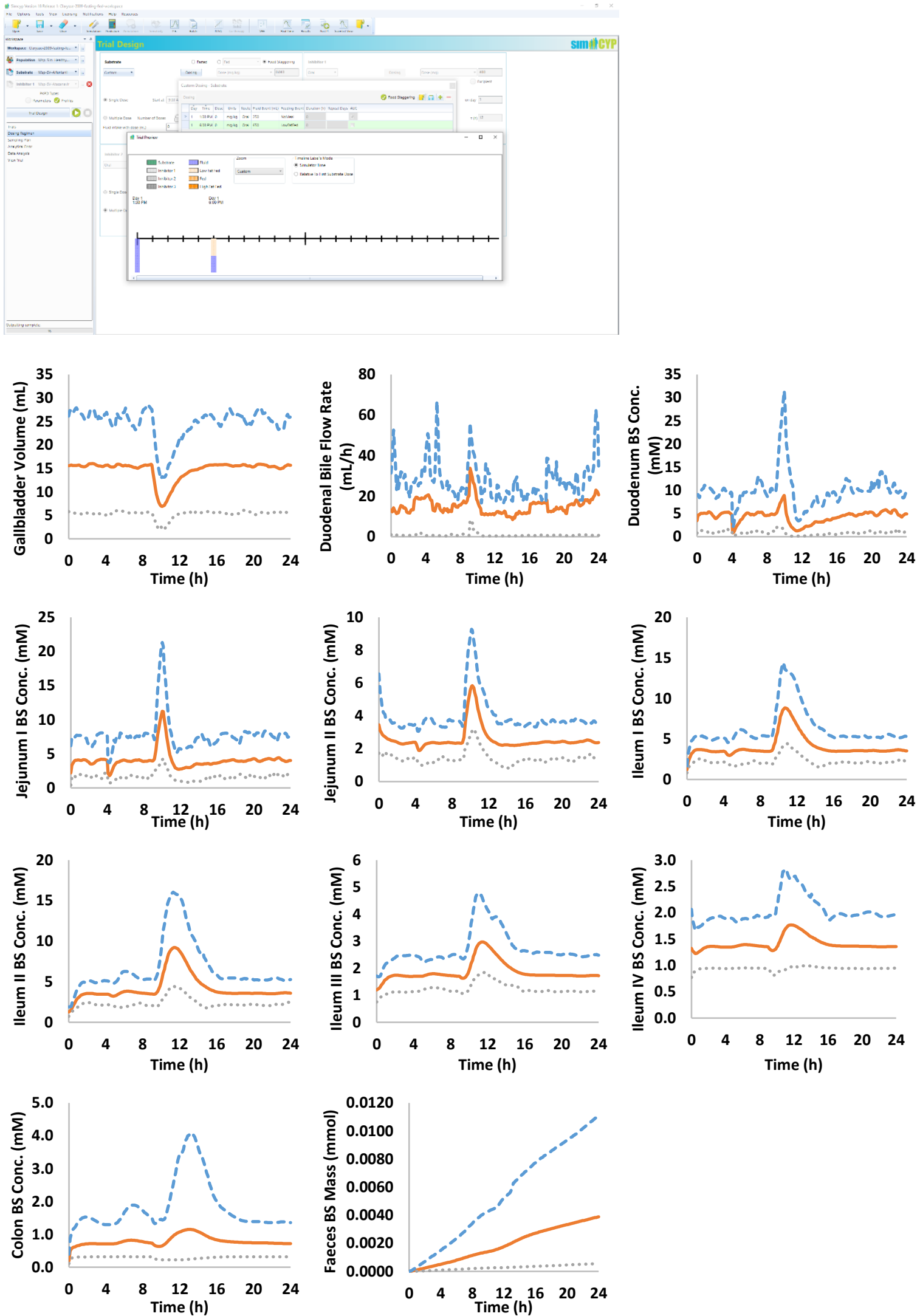
SF2. The image below shows the settings of the trial design in the simulator (Simcyp v18r2) based on Reppas et al 2015 fed protocol and the corresponding ADBSM outputs.
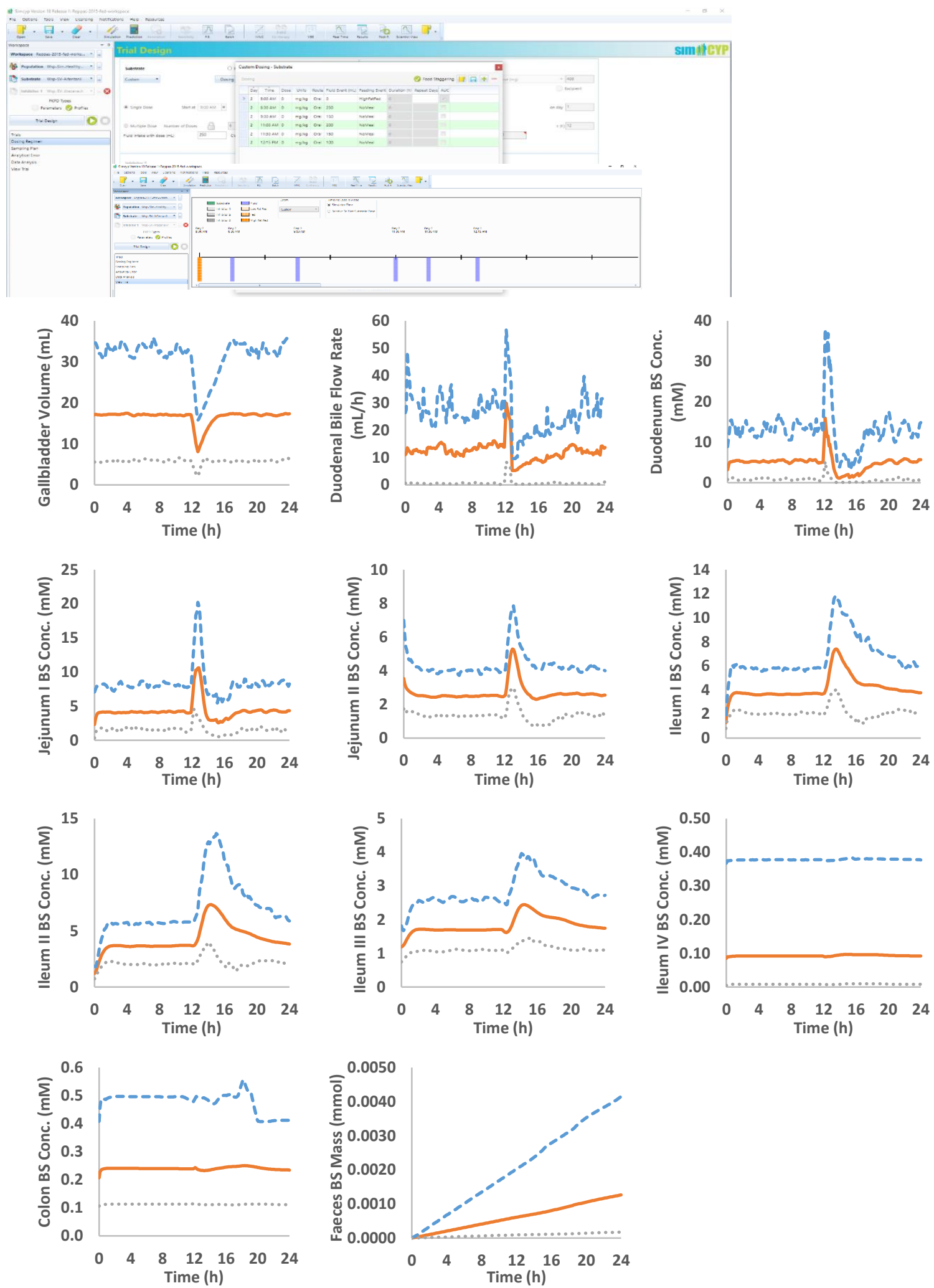
SF3. The image below shows the settings of the trial design in the simulator (Simcyp v18r2) based on Reppas et al 2015 fasting protocol and the corresponding ADBSM outputs.
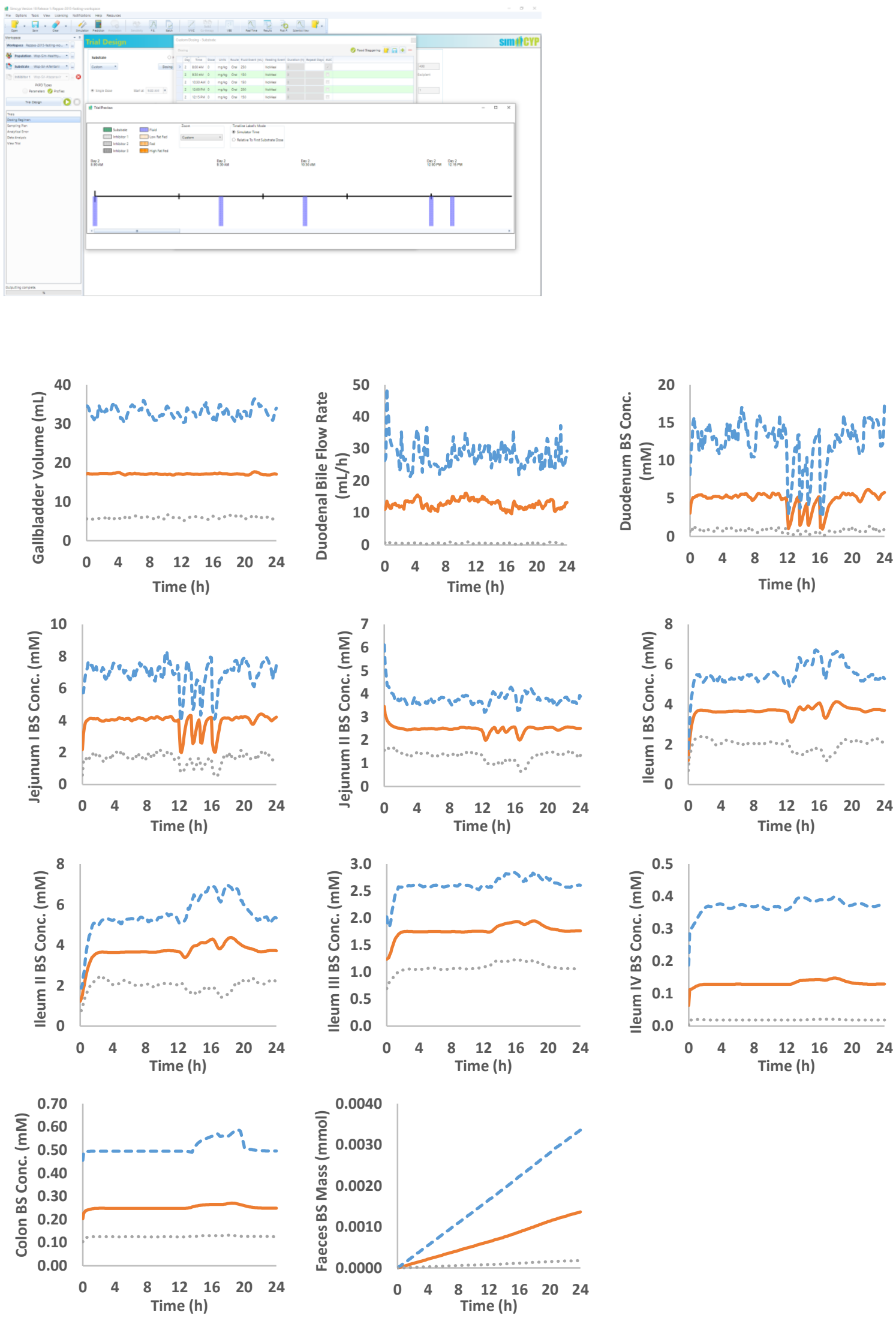
SF4. The image below shows the settings of the trial design in the simulator (Simcyp v18r2) based on Diakidou et al 2009 protocol and the corresponding ADBSM outputs.
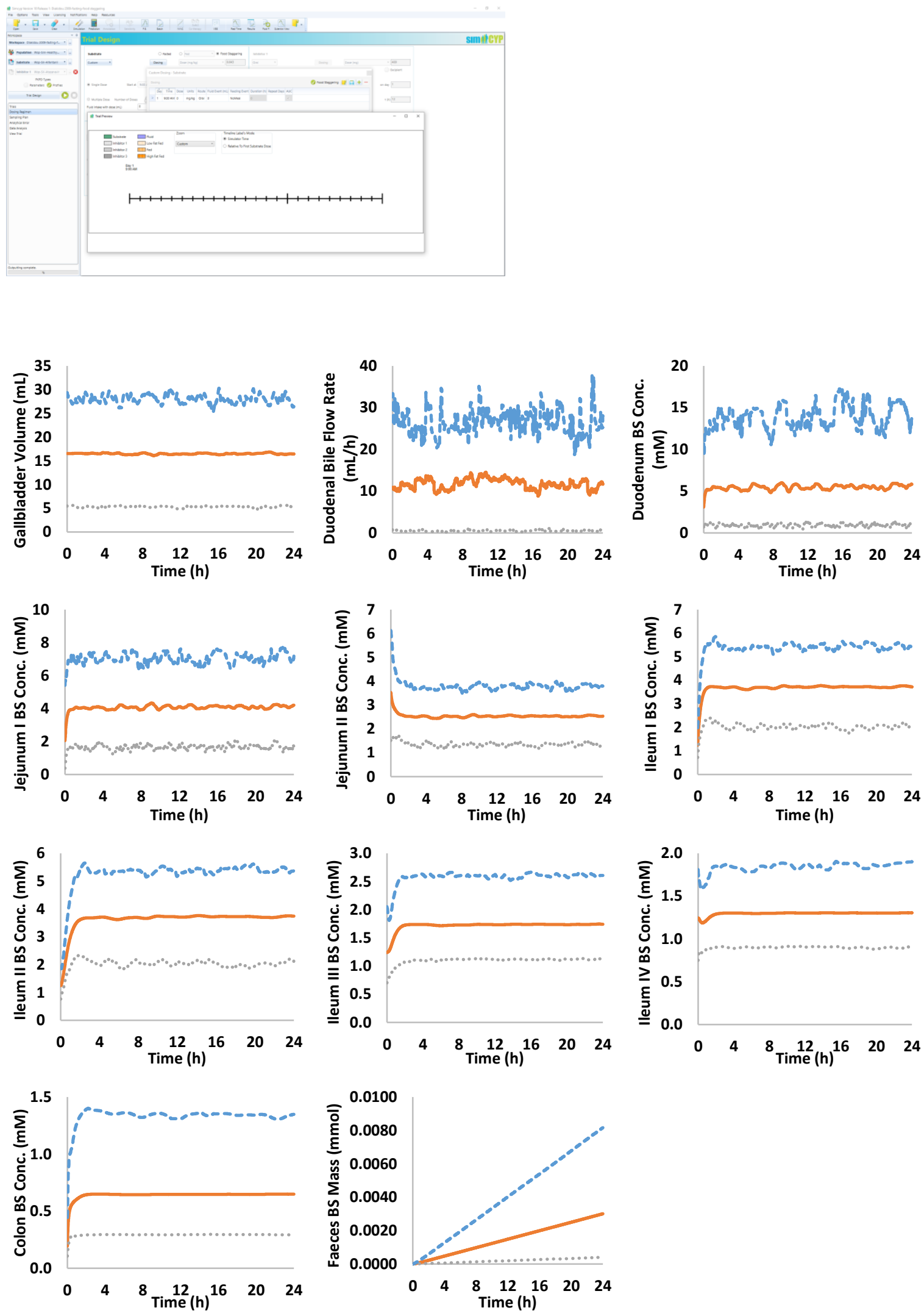
SF5. The image below shows the settings of the trial design in the simulator (Simcyp v18r2) based on Diakidou et al $\mathbf{2 0 0 9}$ protocol and the corresponding ADBSM outputs.
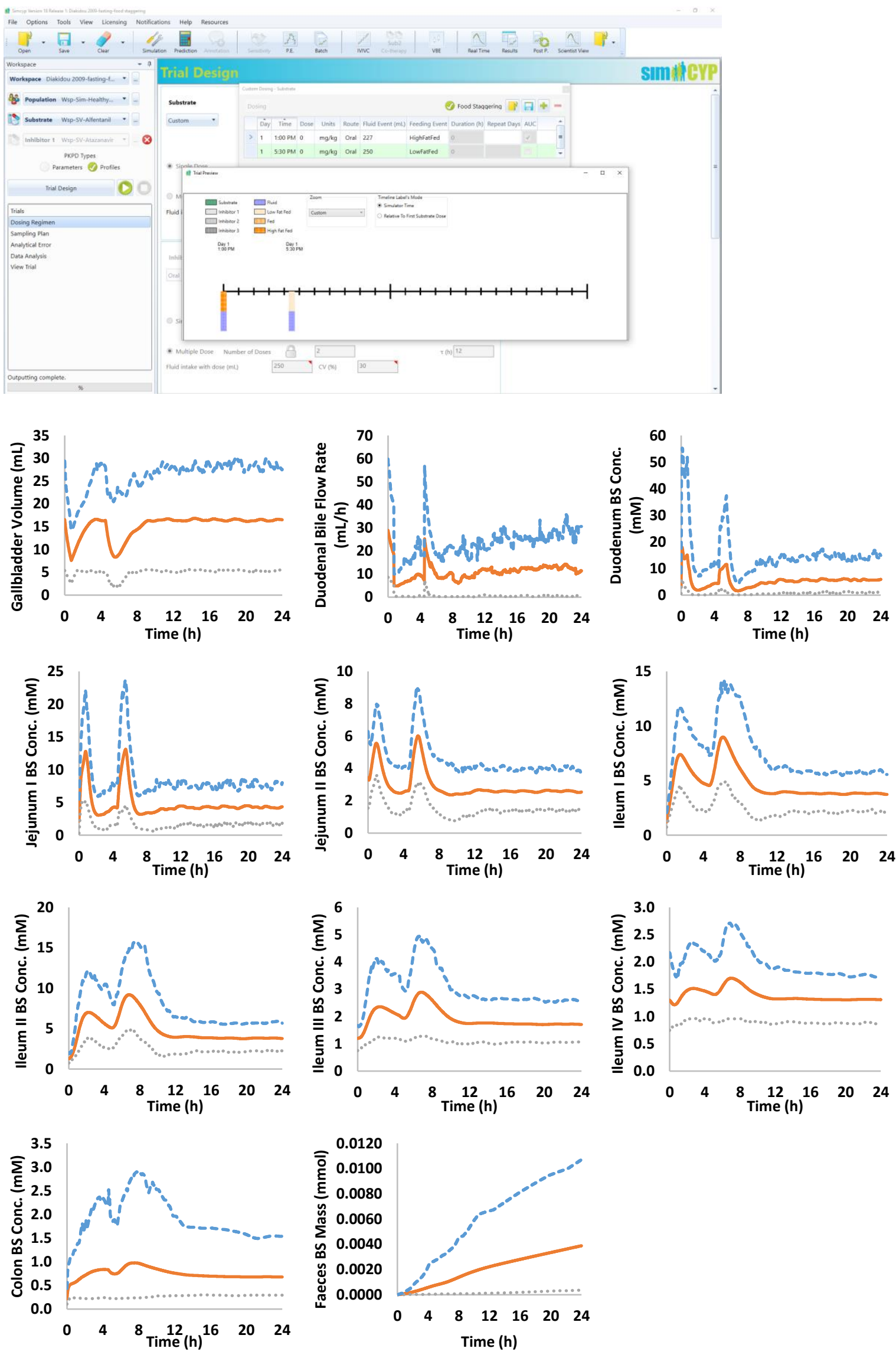
SF6. ADBSM outputs (Simcyp v18r2) based on Yao et al 2002 study
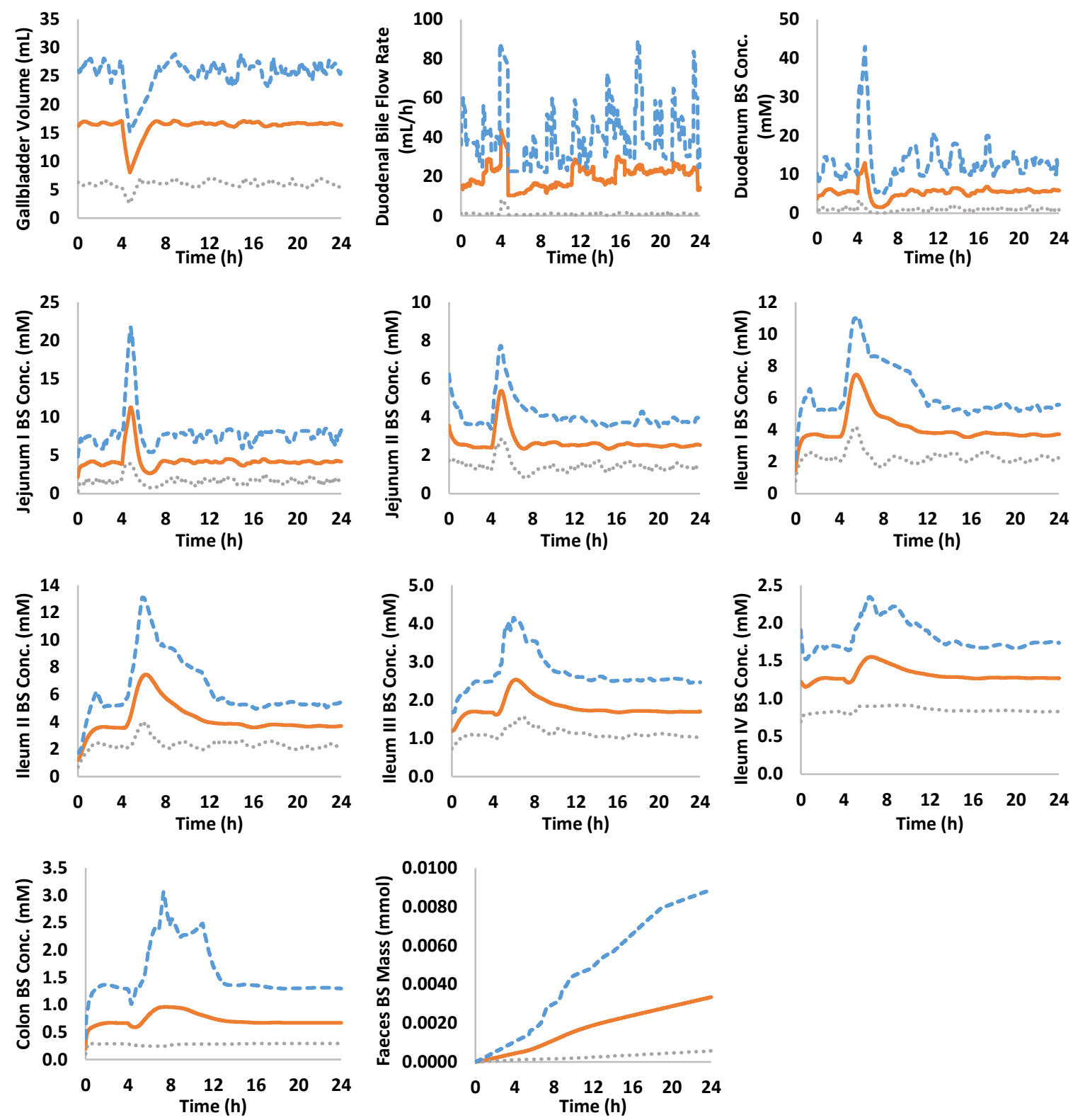
SF7. ADBSM outputs (Simcyp v18r2) based on Hernell et al 1990 study
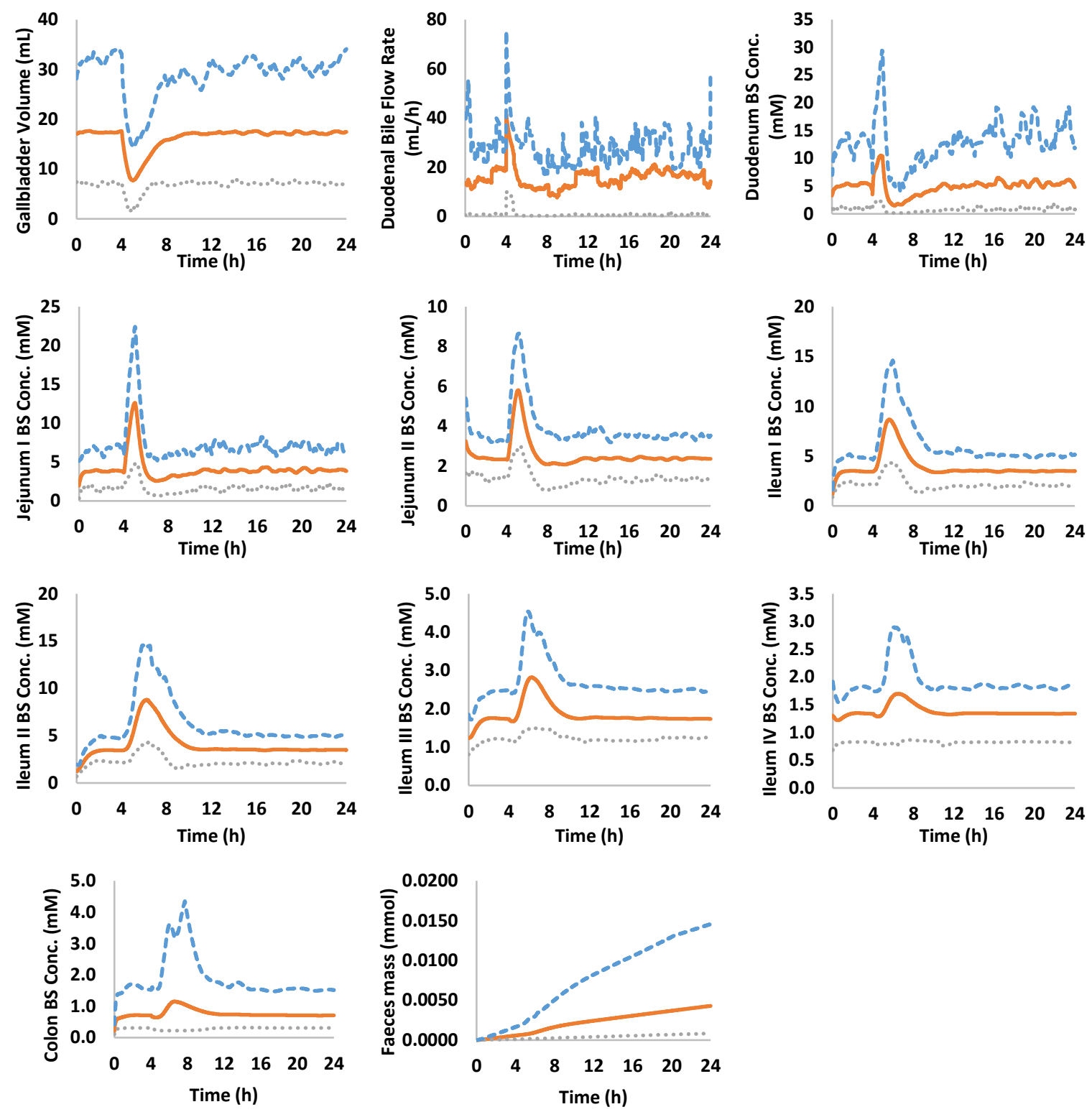
SF8. ADBSM outputs (Simcyp v18r2) based on Meira Fields and H. L. Duthue 1965 clinical study
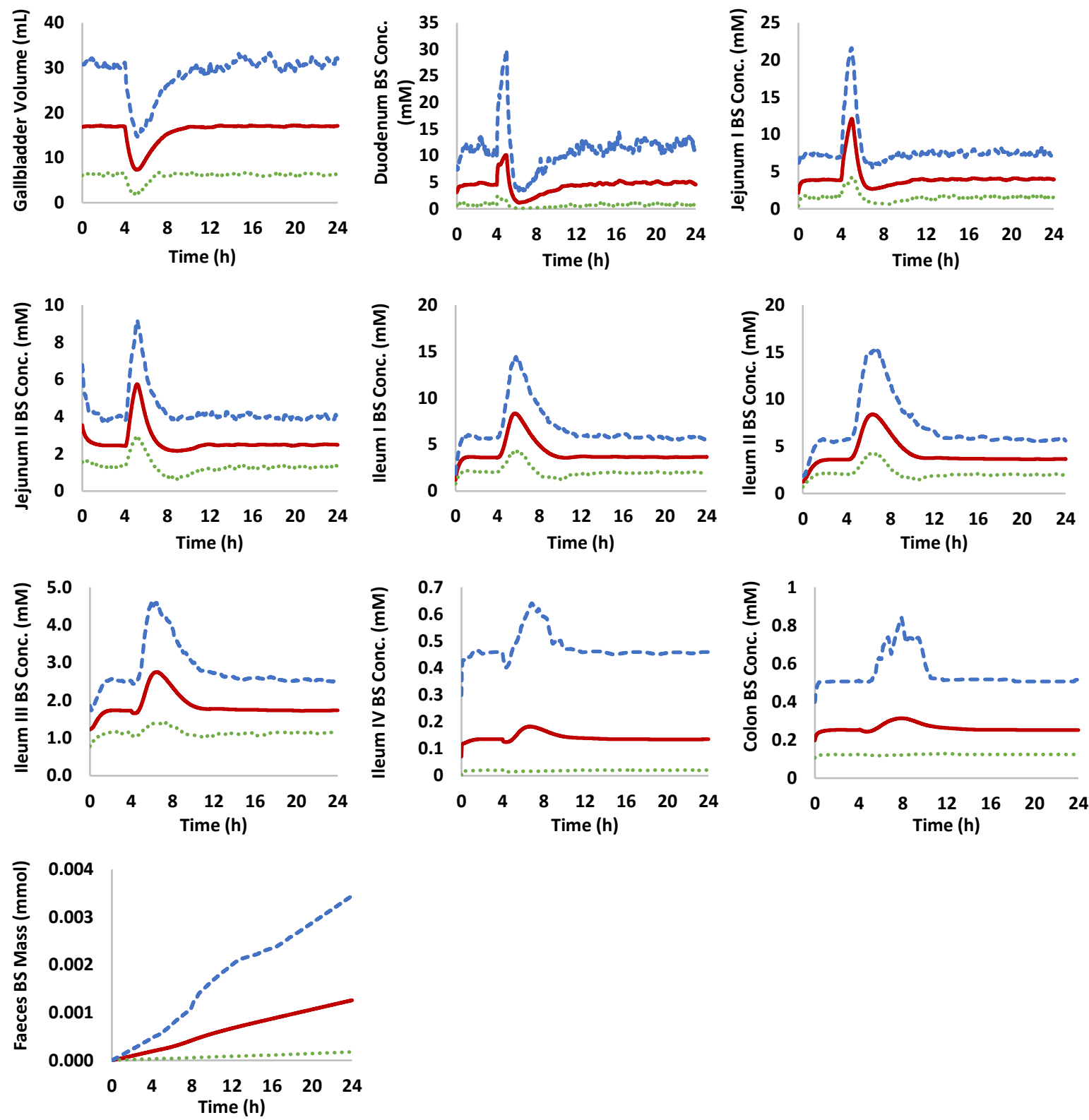
SFig. 9. ADBSM duodenal outputs (Simcyp v18r2) based on Riethorst et al 2016 clinical study

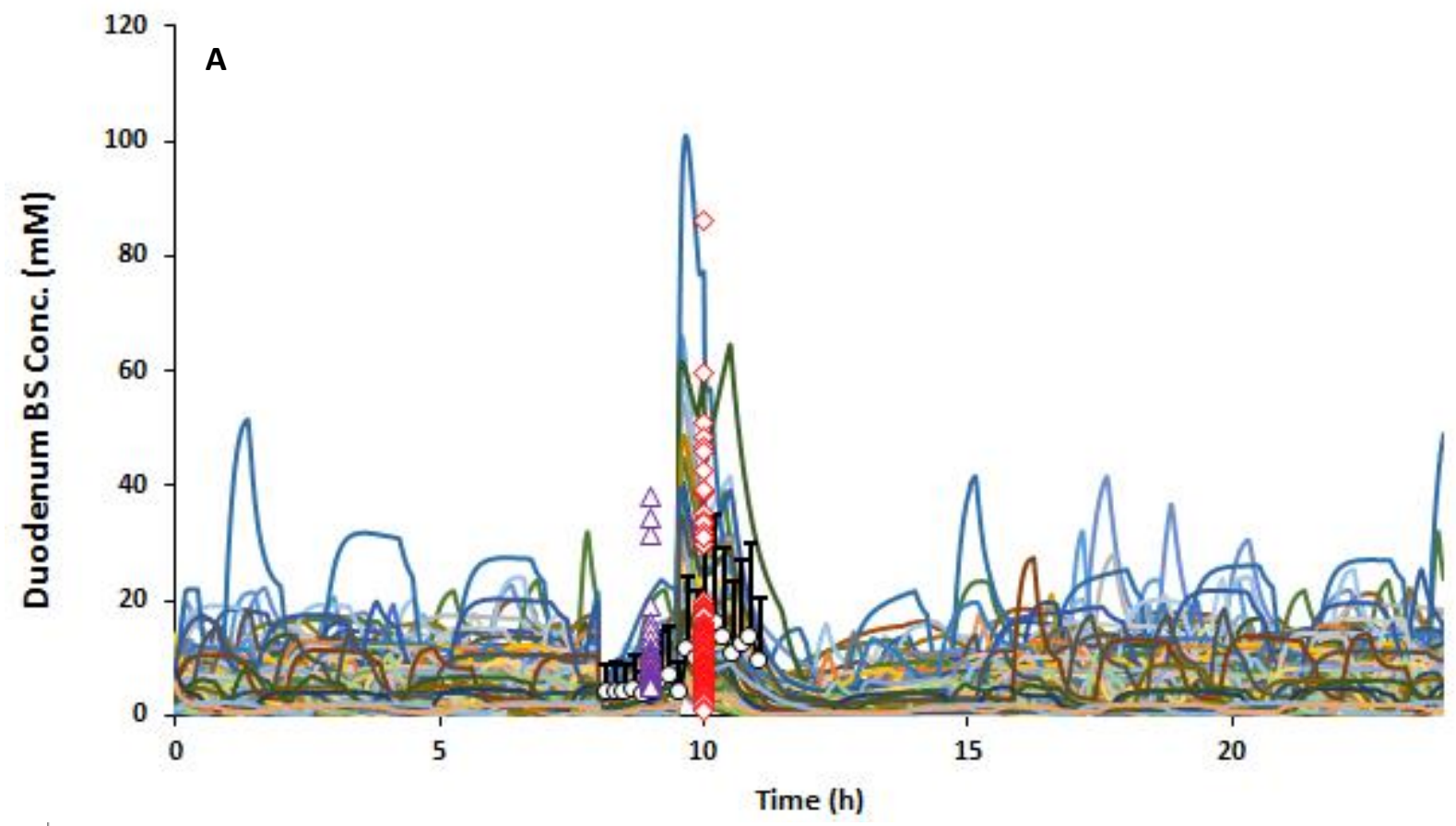

- - Riethorst et al 2016

$\Delta$ Riethorst et al 2016 (fasted

$\diamond$ Riethorst et al 2016 (fed)
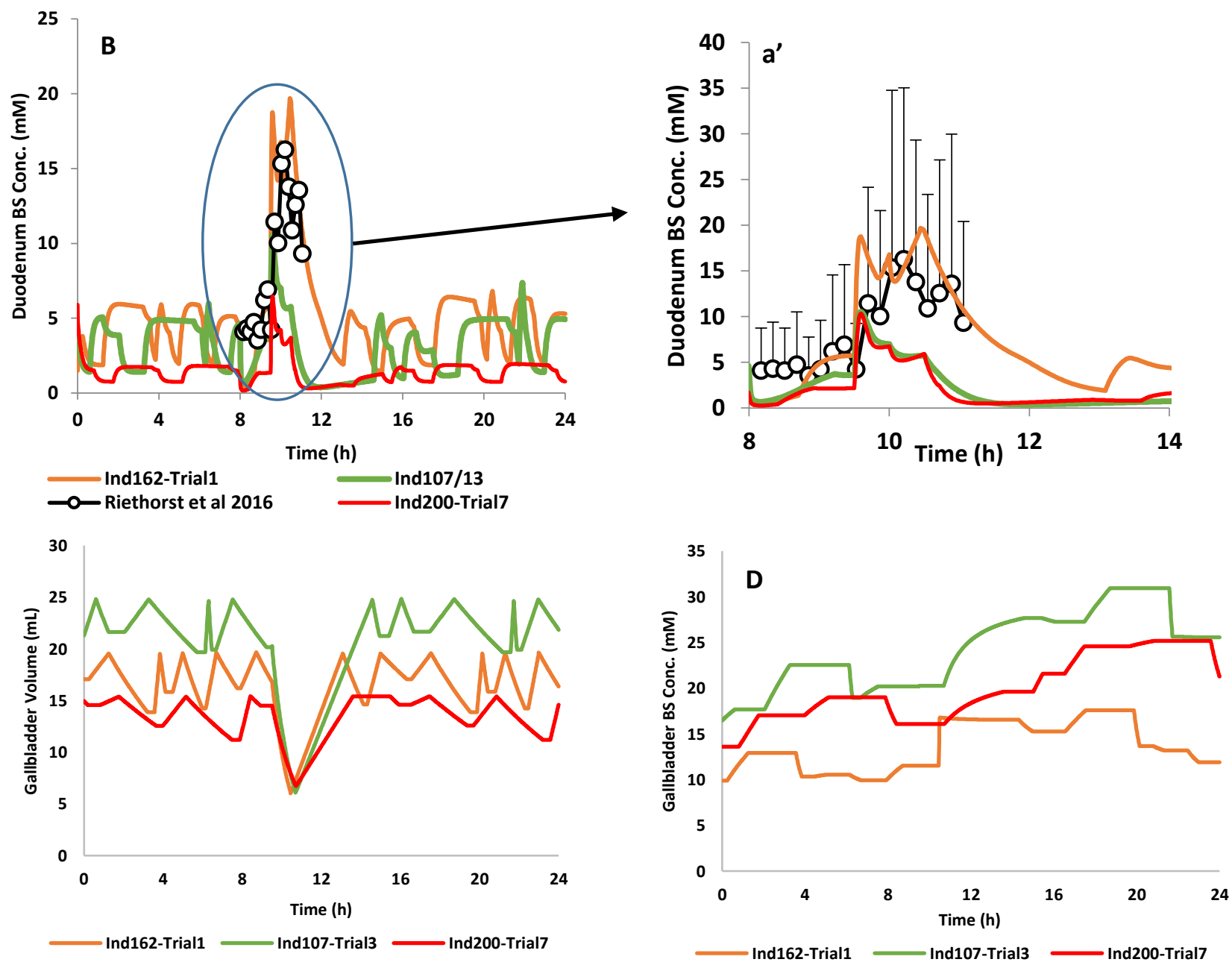
SFig. 9. Riethorst et al 2016 (continued)

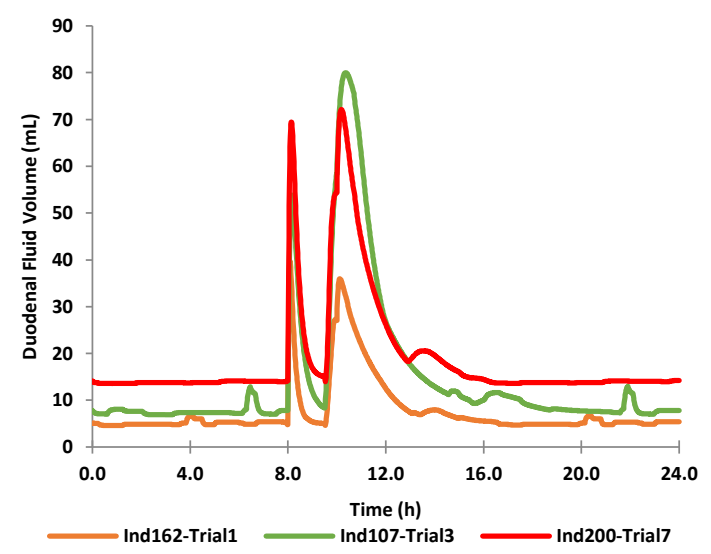

SF10. ADBSM Ileal outputs (Simcyp v18r2) based on Fields \& Duthue 1965 study
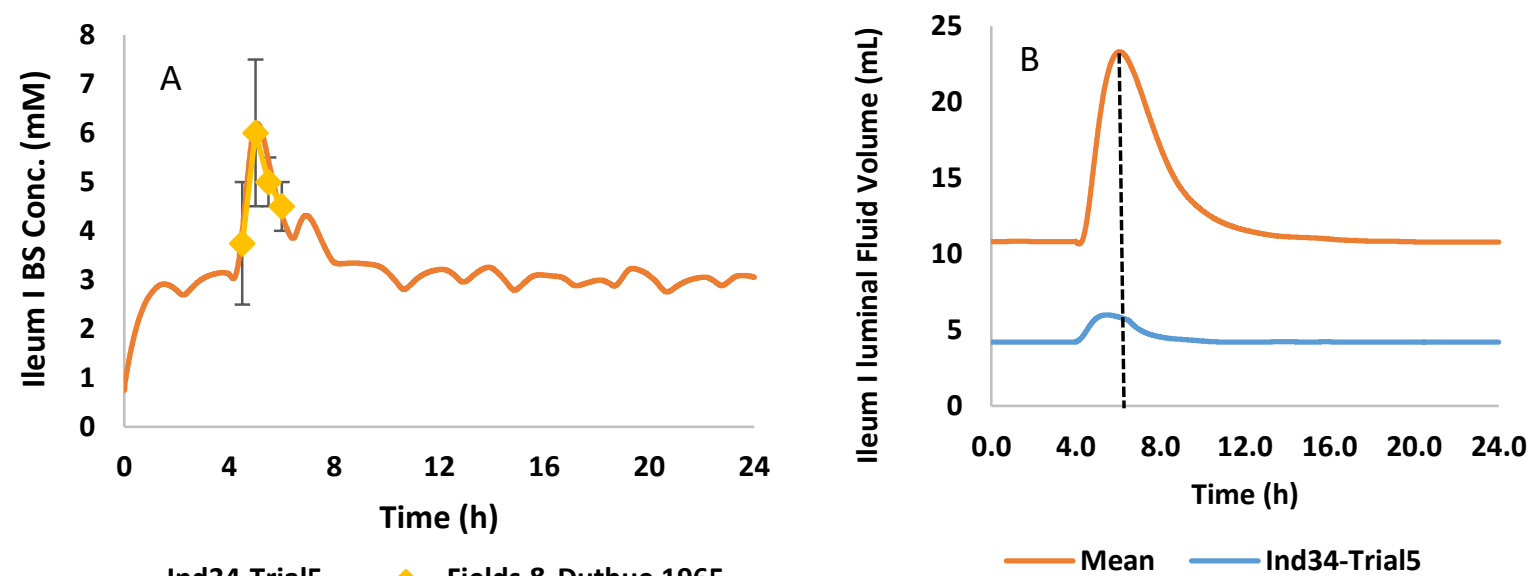
SF11. ADBSM and luminal fluid volume colonic outputs (Simcyp v18r2) based on Diakidou et al 2009 study.
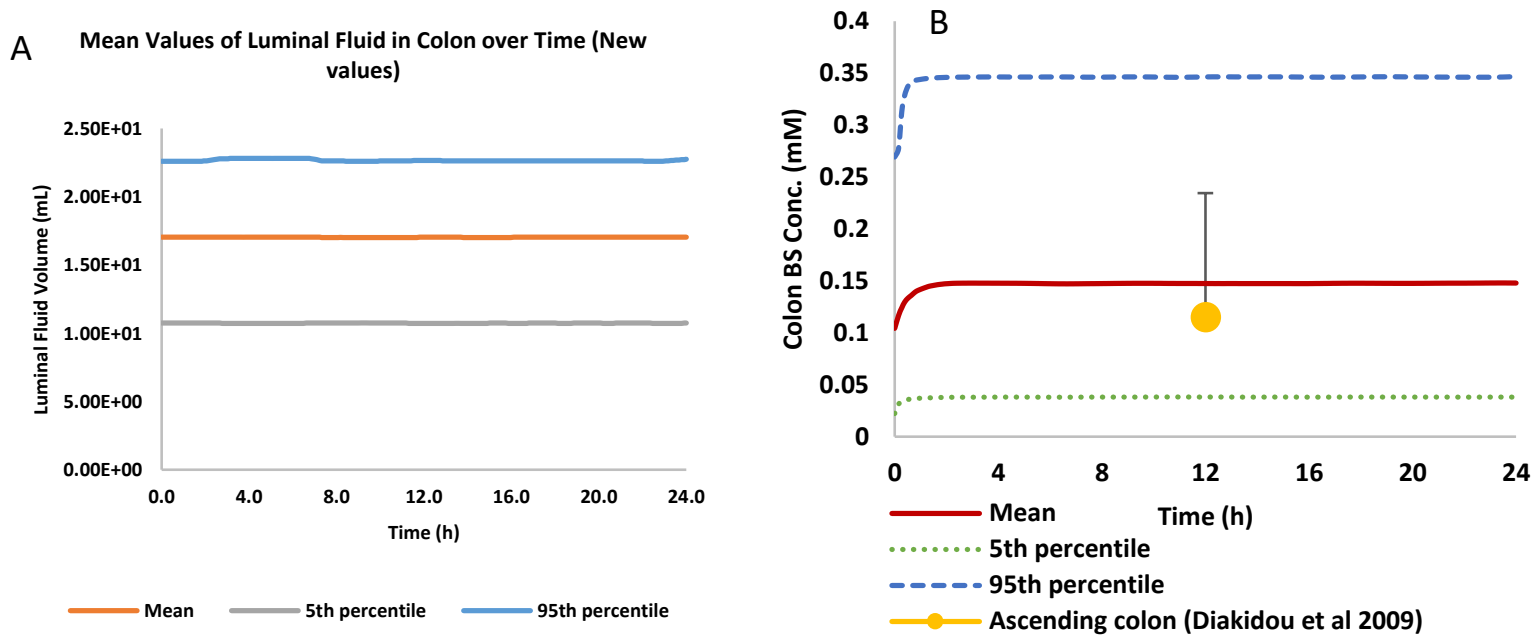

SF12. Relationship between GB' emptying and filling rate constant after a meal

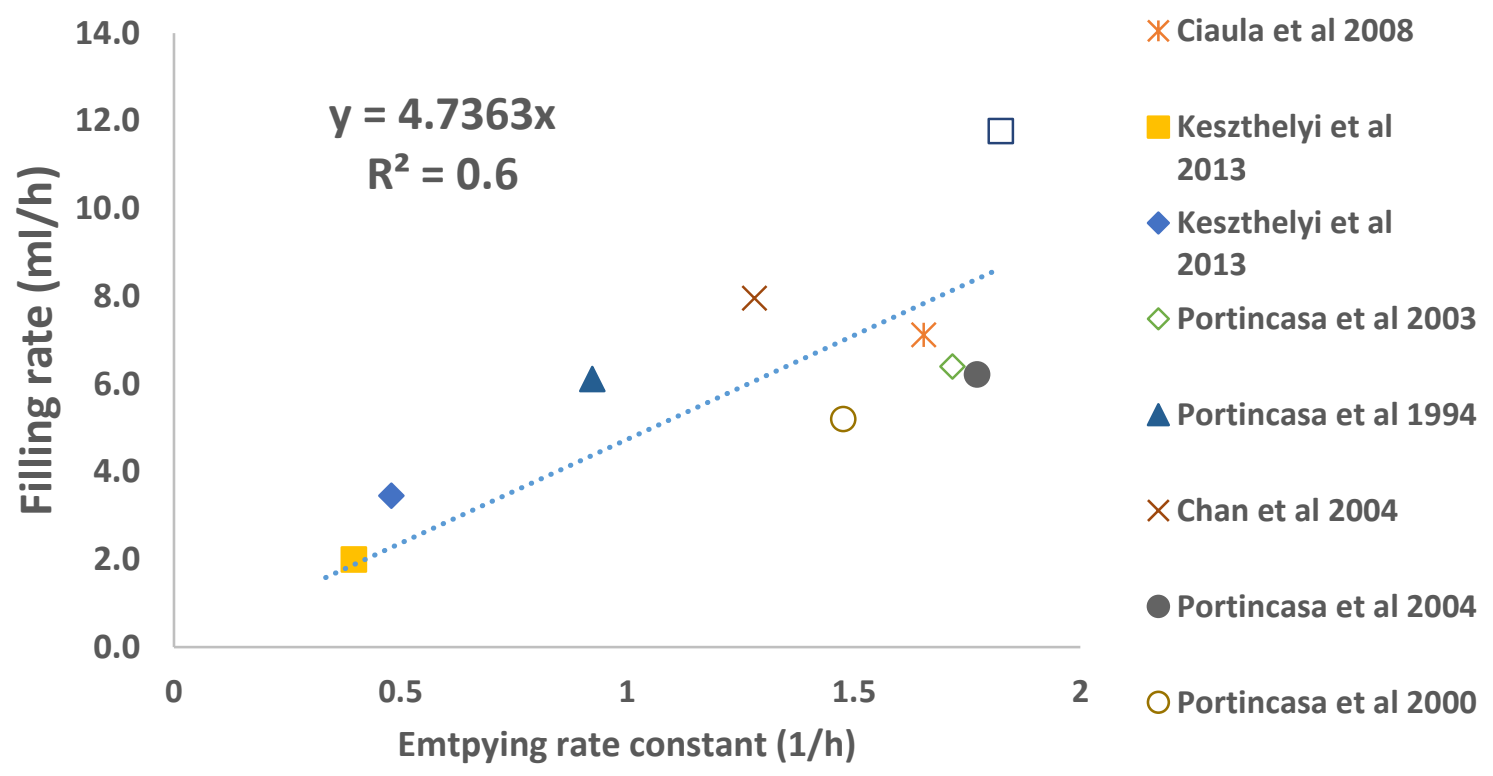




\section{References}

1. Riethorst, D.; Mols, R.; Duchateau, G.; Tack, J.; Brouwers, J.; Augustijns, P. Characterization of Human Duodenal Fluids in Fasted and Fed State Conditions. Journal of Pharmaceutical Sciences 2016, 105, (2), 673-681.

2. Hernell, O.; Staggers, J. E.; Carey, M. C. Physical-chemical behavior of dietary and biliary lipids during intestinal digestion and absorption. 2. Phase analysis and aggregation states of luminal lipids during duodenal fat digestion in healthy adult human beings. Biochemistry 1990, 29, (8), 204156.

3. Di Ciaula, A.; Covelli, M.; Berardino, M.; Wang, D. Q.; Lapadula, G.; Palasciano, G.; Portincasa, P. Gastrointestinal symptoms and motility disorders in patients with systemic scleroderma. $B M C$ Gastroenterology 2008, 8, (1), 7.

4. Keszthelyi, D.; Knol, D.; Troost, F. J.; van Avesaat, M.; Foltz, M.; Masclee, A. A. Time of ingestion relative to meal intake determines gastrointestinal responses to a plant sterol-containing yoghurt drink. European journal of nutrition 2013, 52, (4), 1417-20.

5. Portincasa, P.; Di Ciaula, A.; Baldassarre, G.; Palmieri, V.; Gentile, A.; Cimmino, A.; Palasciano, G. Gallbladder motor function in gallstone patients: sonographic and in vitro studies on the role of gallstones, smooth muscle function and gallbladder wall inflammation. Journal of hepatology 1994, $21,(3), 430-40$.

6. Portincasa, P.; Moschetta, A.; Colecchia, A.; Festi, D.; Palasciano, G. Measurements of gallbladder motor function by ultrasonography: towards standardization. Digestive and Liver Disease 2003, 35, 56-61.

7. Chan, D. C.; Chang, T. M.; Chen, C. J.; Chen, T. W.; Yu, J. C.; Liu, Y. C. Gallbladder contractility and volume characteristics in gallstone dyspepsia. World journal of gastroenterology 2004, 10, (5), 721-4.

8. Portincasa, P.; Peeters, T. L.; van Berge-Henegouwen, G. P.; van Solinge, W. W.; Palasciano, G.; van Erpecum, K. J. Acute intraduodenal bile salt depletion leads to strong gallbladder contraction, altered antroduodenal motility and high plasma motilin levels in humans. Neurogastroenterology and motility : the official journal of the European Gastrointestinal Motility Society 2000, 12, (5), 421-30.

9. Portincasa, P.; Moschetta, A.; Berardino, M.; Di-Ciaula, A.; Vacca, M.; Baldassarre, G.; Pietrapertosa, A.; Cammarota, R.; Tannoia, N.; Palasciano, G. Impaired gallbladder motility and delayed orocecal transit contribute to pigment gallstone and biliary sludge formation in betathalassemia major adults. World journal of gastroenterology 2004, 10, (16), 2383-90.

10. Clarysse, S.; Tack, J.; Lammert, F.; Duchateau, G.; Reppas, C.; Augustijns, P. Postprandial evolution in composition and characteristics of human duodenal fluids in different nutritional states. Journal of Pharmaceutical Sciences 2009, 98, (3), 1177-1192.

11. Pedersen, B. L.; Mullertz, A.; Brondsted, H.; Kristensen, H. G. A comparison of the solubility of danazol in human and simulated gastrointestinal fluids. Pharmaceutical research 2000, 17, (7), 891-4.

12. Perez de la Cruz Moreno, M.; Oth, M.; Deferme, S.; Lammert, F.; Tack, J.; Dressman, J.; Augustijns, P. Characterization of fasted-state human intestinal fluids collected from duodenum and jejunum. The Journal of pharmacy and pharmacology 2006, 58, (8), 1079-89.

13. Fields, M.; Duthie, H. L. Effect of vagotomy on intraluminal digestion of fat in man. Gut 1965, 6, (3), 301-310.

14. Reppas, C.; Karatza, E.; Goumas, C.; Markopoulos, C.; Vertzoni, M. Characterization of Contents of Distal lleum and Cecum to Which Drugs/Drug Products are Exposed During Bioavailability/Bioequivalence Studies in Healthy Adults. Pharmaceutical research 2015, 32, (10), 3338-3349. 
15. Diakidou, A.; Vertzoni, M.; Goumas, K.; Söderlind, E.; Abrahamsson, B.; Dressman, J.; Reppas, C. Characterization of the Contents of Ascending Colon to Which Drugs are Exposed After Oral Administration to Healthy Adults. Pharmaceutical research 2009, 26, (9), 2141-2151.

16. Yao, L.; Heubi, J. E.; Buckley, D. D.; Fierra, H.; Setchell, K. D.; Granholm, N. A.; Tso, P.; Hui, D. Y.; Woollett, L. A. Separation of micelles and vesicles within lumenal aspirates from healthy humans: solubilization of cholesterol after a meal. Journal of lipid research 2002, 43, (4), 654-60.

17. Kalantzi, L.; Goumas, K.; Kalioras, V.; Abrahamsson, B.; Dressman, J. B.; Reppas, C. Characterization of the human upper gastrointestinal contents under conditions simulating bioavailability/bioequivalence studies. Pharmaceutical research 2006, 23, (1), 165-76. 